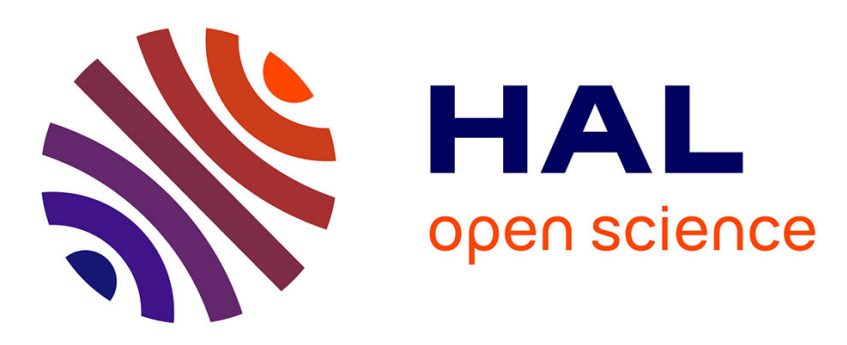

\title{
Influence of viscosity on entropy noise generation through a nozzle
}

Maxime Huet, Ariane Emmanuelli, Sebastien Ducruix

\section{To cite this version:}

Maxime Huet, Ariane Emmanuelli, Sebastien Ducruix. Influence of viscosity on entropy noise generation through a nozzle. Journal of Sound and Vibration, 2021, 510, pp.116293. 10.1016/j.jsv.2021.116293 . hal-03279279

\section{HAL Id: hal-03279279 \\ https://hal.science/hal-03279279}

Submitted on 6 Jul 2021

HAL is a multi-disciplinary open access archive for the deposit and dissemination of scientific research documents, whether they are published or not. The documents may come from teaching and research institutions in France or abroad, or from public or private research centers.
L'archive ouverte pluridisciplinaire HAL, est destinée au dépôt et à la diffusion de documents scientifiques de niveau recherche, publiés ou non, émanant des établissements d'enseignement et de recherche français ou étrangers, des laboratoires publics ou privés. 


\title{
Influence of viscosity on entropy noise generation through a nozzle
}

\author{
Maxime Huet ${ }^{\mathrm{a}, *}$, Ariane Emmanuelli ${ }^{\mathrm{a}, 1}$, Sébastien Ducruix ${ }^{\mathrm{b}}$ \\ ${ }^{a} D A A A$, ONERA, Université Paris Saclay, F-92322 Châtillon, France \\ ${ }^{b}$ Laboratoire EM2C, CNRS, CentraleSupélec, Université Paris-Saclay, 3, Rue Joliot Curie, \\ 91192, Gif-sur-Yvette Cedex, France
}

\begin{abstract}
Entropy (temperature) fluctuations produced by turbulent flames generate noise when they are accelerated by the flow. This so-called entropy noise is an important contributor to core noise in modern aeroengines and several semi-analytical models exist in the litterature for its prediction. All these models assume the flow to be inviscid. In the present paper, contribution of viscosity on entropy noise generation and scattering through a nozzle is investigated numerically with URANS simulations and analytically through the extension of the 2D inviscid model of Emmanuelli et al. (Journal of Sound and Vibration 472:115163 (2020)). Simulations indicate noise generation and scattering is slightly reduced in the medium-frequency range in the presence of viscosity with variations below $3 \mathrm{~dB}$ in comparison to reference inviscid data. This noise variation is qualitatively well reproduced by the low-order model. The major effect of viscosity on noise generation and propagation lies in the presence of boundary layers. Viscous entropy noise sources and viscous diffusion of acoustic perturbations have a negligible impact on noise. Discrepancies between simulations and analytical solutions are found to come from the radial evolution of the acoustic waves in thick boundary layers, not accounted for in the model, and which impact noise

\footnotetext{
* Corresponding author

Email address: maxime.huet@onera.fr (Maxime Huet)

${ }^{1}$ now working at Physics of Fluids Group, Max Planck Center Twente for Complex Fluid Dynamics, J. M. Burgers Center for Fluid Dynamics and MESA+ Research Institute, University of Twente, P. O. Box 217, 7500 AE Enschede, The Netherlands
}

Preprint submitted to Journal of Sound and Vibration

June 24, 2021 
scattering.

Keywords: entropy noise, low-order model, nozzle, viscosity

\section{Introduction}

The important efforts carried out during the last decades to reduce jet and fan noise in turbojet engines led to the emergence of additional noise sources, previously masked, among which combustion noise stands out [1, 2, Combus-

5 tion noise issues are even more important for turboshaft engines where jet and fan noises are absent [3, 4. Two different mechanisms are involved in combustion noise. Direct combustion noise is associated with the acoustic fluctuations generated by the turbulent flame through heat release fluctuations [5, 6], whereas indirect combustion noise is produced when flow heterogeneities, such as entropy (cold and hot spots), vorticity (turbulent spots) and compositional (mixture heterogeneities) fluctuations are accelerated by the mean flow through nozzles and turbine stages $7-10$. Such perturbations lead to a loss of balance, to which the flow reacts by emitting acoustic waves [11, 12. Relative contributions of direct and indirect combustion noise to global sound emission of aeroengines is still an open question [1, 13] but recent analytical investigations suggest indirect noise may dominate direct noise in several practical situations [1, 14, hence the need for its modelling and reduction. Concerning indirect noise sources, entropy noise is thought to dominate vorticity noise because of the large entropy fluctuations produced by the turbulent flames inside combustion chambers, as well as large dissipation of turbulent structures by viscosity, particularly important at high temperature [15]. As for compositional noise, it was shown analytically to be a possible contributor in lean mixtures and supercritical nozzle flow regimes only [16, 17].

The observations above outline the need for modelling of entropy noise. The present paper focuses on its generation in a nozzle. Nozzle flow is a simple configuration very well suited to the investigation of entropy noise through analytical, numerical and experimental approaches, before possible application 
to more realistic turbine geometries. Analytical studies dedicated to entropy noise in nozzles essentially build on the seminal work of Marble and Candel 18 assuming one-dimensional flow (flow variables do not vary along radial and azimuthal directions). Noise produced and scattered through the nozzle is expressed in the form of transfer functions evaluated algebraically in the compact limit, i.e. acoustic and entropic wavelengths are assumed large compared to the nozzle dimensions. Non-compact transfer functions are also evaluated in the simplified configuration of a linear velocity profile, an hypothesis later relaxed by Moase et al. [19] and Giauque et al. 20] considering a piecewise-linear velocity profile. Using a different approach, Bohn [21] solves a system of partial differential equations over pressure, velocity and entropy to determine the transfer functions of generic nozzles and provides asymptotic solutions in the 40 large frequency limit in the case of a linear velocity profile, whereas Duran and Moreau 22] consider a system of partial differential equations over mass flow rate, stagnation temperature and entropy solved with the Magnus expansion. Additionally, Mahmoudi et al. 23] describe the nozzle geometry as a succession of ducts of constant radii and apply the compact solutions of Marble and Candel between ducts to reconstruct frequency-dependant transfer functions. The one-dimensional hypothesis was recently relaxed by Duran and Morgans 24, Dowling and Mahmoudi [1] and Mahmoudi et al. 23] by considering azimuthal flow fluctuations in annular nozzles and by Emmanuelli et al. [25] and Huet et al. 26] to account for the radial evolution of the flow. The former studies allow to investigate the contribution of the different azimuthal modes in indirect noise, whereas the latter addresses the problem of shear dispersion of entropy fluctuations by the mean flow and subsequent noise modifications. The role of entropy dispersion in indirect noise production is also addressed in a different way by Mahmoudi et al. [27] in a combustion chamber. The authors convect entropy fluctuations along streamlines and reconstruct a radially averaged entropy fluctuation at combustor outlet, used to feed a one-dimensional model for the nozzle. To end, the isentropic nozzle assumption is considered by De Domenico et al. 28. Marble and Candel's [18] model is adapted to non isentropic flows, 
corresponding for instance to the presence of recirculation, and compact transfer functions are expressed as a function of a pressure loss parameter.

The hypotheses used in the models may appear restrictive in comparison with the complex phenomena occurring in real flows. Concerning acoustics, it is often argued that waves cannot exhibit radial variations because corresponding radial modes are cut-off for the low frequencies considered and viscosity can be neglected due to the short propagation distance. Limited contribution of acoustic-boundary layer interactions also plays in favour of the one-dimensional flow assumption. Duran and Moreau 22] and Huet [29] showed that such onedimensional models indeed capture the acoustic reflection and transmission coefficients of subsonic and choked nozzle flows with a good accuracy. The compact 70 model is also suitable to capture entropy noise in the compact limit, as illustrated by Leyko et al. [30] with the reproduction of the experimental Entropy Wave Generator test case in choked configuration [31. When larger frequencies are considered, Morgans et al. 32 and Giusti et al. 33. numerically demonstrated that turbulent dissipation of entropy is negligible for constant section duct flows. This finding was also observed through a nozzle flow by Becerril [34 and Moreau et al. 35. Common to these studies is the importance of shear dispersion of entropy perturbations by the mean flow and possible contribution of turbulent mixing in entropy dispersion, the latter being restricted to large frequencies because of the limited diameter of the duct where only small-scale turbulence can develop. Inside the combustion chamber, however, large turbulent structures may grow and contribute to the dispersion of entropy fluctuations before they reach the nozle, as demonstrated by Xia et al. 36 in a realistic gas turbine combustor flow-field. Additionally, thermal diffusion may play a role in the attenuation of entropy fluctuations. It was found numerically to be negligible by Xia et al. [36] in the Siemens SGT-100 gas turbine combustor. From a theoretical point of view, thermal diffusion drops with pressure and is expected to be negligible in industrial, pressurized combustors but may contribute to entropy damping in ambient pressure, lab scale experiments [37. Finally, most of the models use the strong assumption of negligible vorticity. 
Entropy-induced vorticity originates from a baroclinic torque when density (entropy) fluctuations are misaligned with the mean pressure gradient [24] and was observed numerically by several authors. Its contribution was shown to be minor for inviscid nozzle flows by Duran and Morgans [24] and Emmanuelli et al. [25] but a potential noise generator when viscosity is considered [34, 35, 38. In addition, viscosity influences the mean flow development, in particular through the presence of boundary layers, and may affect entropy dispersion and noise generation in comparison to inviscid configurations.

The objective of the present paper is to assess the role of viscosity in entropy noise generation within a nozzle through analytical modelling and numerical simulations with the URANS approach. This numerical modelling is sufficient to capture the influence of viscosity on the mean flow, in particular boundary layers, as well as on the propagation of acoustics and convection of entropy and vorticity perturbations. These phenomena are expected to be the dominant ones in the frequency bandwidth of indirect combustion noise considered here, below $1 \mathrm{kHz}$. The interaction between these perturbations and small-scale turbulence is not directly computed and cannot be accounted for in the present simulations, but it is expected to be of second order following literature results [29, 39]. In addition, URANS already proved successful in simulating entropy convection and related noise production through nozzles and turbine stages [31, 39, 41]. The paper is organized as follows. Derivation of the Riemann invariants in duct flows with boundary layers is first detailed in Section 2 under simplifying assumptions. The inclusion of viscous terms in a two-dimensional low-order model is presented in Section 3. This modelling allows for an analytical discussion on the role of viscosity in entropy noise generation. The geometry considered to evaluate the transfer functions is detailed in Section 4, along with the description of the mean flow fields and the numerical procedure to compute the transfer functions with Euler and URANS equations. Modelled and simulated transfer functions are then compared in Section 5 . The role of viscosity in scattering and generation of acoustic waves is discussed for both approaches and the accuracy and limitations of the model are outlined, before providing conclusions in Section 6 . 


\section{Planar acoustic waves in straight duct}

Both low-order modelling and numerical simulations require the separation of upstream and downstream travelling acoustic waves in ducts of constant radius upstream and downstream of a nozzle in order to evaluate the transfer functions of the system. This separation is straightforward for one-dimensional flows where the acoustic characteristic waves are obtained with the Riemann invariants. In this section, the expression of the Riemann invariants is extended to the case of two-dimensional duct flows with simplifying hypotheses. The mean flow is assumed to be axisymmetric, oriented in the $x$ direction and invariant with respect to this coordinate. Tangential velocity is neglected (non-swirling flow). Continuity and axial momentum equations write, in cylindrical coordinates

$$
\begin{aligned}
\frac{\partial \rho}{\partial t}+\frac{\partial \rho u_{x}}{\partial x}+\frac{\partial \rho u_{r}}{\partial r}+\frac{\rho u_{r}}{r} & =0 \\
\frac{\partial u_{x}}{\partial t}+u_{x} \frac{\partial u_{x}}{\partial x}+u_{r} \frac{\partial u_{x}}{\partial r} & =-\frac{1}{\rho} \frac{\partial p}{\partial x}
\end{aligned}
$$

where $\rho, u_{x}, u_{r}, p, t, x$ and $r$ stand respectively for the density, axial and radial velocities, pressure, time and axial and radial coordinates. The flow variables are then decomposed as a sum of their steady state and perturbation, $p=p_{0}+p^{\prime}$, etc. Perturbations are assumed to be linear and of acoustic nature only with planar acoustic waves, i.e. $\partial p^{\prime} / \partial r=0, \partial u_{x}^{\prime} / \partial r=0$ and $u_{r}^{\prime}=0$. Noticing that mean flow invariance with respect to $x$ coordinate imply $u_{0 r}=0$, the equations above reduce to

$$
\begin{aligned}
\frac{\partial p^{\prime}}{\partial t}+\rho_{0} c_{0}^{2} \frac{\partial u_{x}^{\prime}}{\partial x}+u_{0 x} \frac{\partial p^{\prime}}{\partial x} & =0 \\
\frac{\partial u_{x}^{\prime}}{\partial t}+u_{0 x} \frac{\partial u_{x}^{\prime}}{\partial x} & =-\frac{1}{\rho_{0}} \frac{\partial p^{\prime}}{\partial x}
\end{aligned}
$$

with $c_{0}=\sqrt{\gamma p_{0} / \rho_{0}}$ the mean sound velocity. Multiplying Eq. (4) by $\pm \rho_{0} c_{0}$ and after addition of Eq. (3), it comes

$$
\frac{\partial}{\partial t}\left(p^{\prime} \pm \rho_{0} u_{0} u_{x}^{\prime}\right)+\left(u_{0 x} \pm c_{0}\right) \frac{\partial}{\partial x}\left(p^{\prime} \pm \rho_{0} u_{0} u_{x}^{\prime}\right)=0
$$


Equation (5) corresponds to the propagation equation of the fluctuation $p^{\prime} \pm$ $\rho_{0} c_{0} u_{x}^{\prime}$ at the velocity $u_{0 x} \pm c_{0}$. In the practical configurations considered hereafter the mean flow may vary slowly along the axial direction, the derivation above is therefore not exact but it is considered to be a good approximation. The classical 1D impedance relations therefore remain accurate even for 2D duct flows and the non-dimensional form of the acoustic waves inside the duct may write

$$
P^{ \pm}=\frac{1}{2}\left(\frac{p^{\prime}}{\gamma \bar{p}_{0}} \pm \frac{\overline{\rho_{0} c_{0}}}{\gamma \bar{p}_{0}} u_{x}^{\prime}\right)
$$

where the overlined quantities $\overline{(\cdot)}$ are reduced to 1D quantities using Eq. (7), with $A$ the duct section. It is worth mentioning that $\overline{p^{\prime}}=p^{\prime}$ and $\overline{u_{x}^{\prime}}=u_{x}^{\prime}$ for planar acoustic waves.

$$
\bar{f}=\frac{1}{A} \int_{A} f d A
$$

\section{2D model}

In this section, the two-dimensional model proposed by Emmanuelli et al. 25] for Euler flows is extended to include viscosity effects. This model is based on the mass conservation and axial momentum equations. Mass conservation is not impacted by viscosity, which only appears in the momentum equation. The derivation of the filtered momentum equation in cylindrical coordinates is detailed in Appendix A and takes a similar form for URANS, LES and DNS. The difference between these three numerical modelling methods lies in the value of the viscosity. In DNS, all the physical scales are solved on the numerical grid with a proper time step and viscosity corresponds to the molecular viscosity $\mu$. In LES, small scale turbulence cannot be solved on the mesh and its associated dissipation is modelled through an eddy viscosity coefficient $\mu_{T}$, leading to an effective viscosity $\mu_{T}+\mu$. Turbulence is completely modelled in URANS through the eddy viscosity term, which exhibits values much larger than in LES. The kinetic energy per unit volume of the modelled fluctuations $k$ also appears in the momentum equation but it is often neglected 42 . 
The model obeys the following hypotheses.

- The nozzle is oriented along the $x$ axis and the fluid flows towards the

165

170

to URANS where these terms encompass all the flow turbulence. From this point of view, application of the model with RANS flow fields could lead to a better simulation of a key part of the physical phenomena.

\subsection{Equations of the model}

Viscosity does not affect the mass conservation equation, which is identical to the inviscid case. It is obtained by application of a mass budget on a slice of the nozzle between positions $x$ and $x+d x$ and writes

$$
\frac{\partial \bar{\rho}}{\partial t}+\frac{\partial \overline{\rho u_{x}}}{\partial x}=-\frac{1}{A} \overline{\rho u_{x}} \frac{\mathrm{d} A}{\mathrm{~d} x}
$$


where the symbols for Reynolds- and Favre-averaged quantities are dropped to simplify the notations and the overlined quantities correspond to the $1 \mathrm{D}$ averaging of Eq. (7). Following the hypotheses listed above, the axial momentum equation reduces to (see Appendix A and Hoffman et al. [43])

$$
\frac{\partial u_{x}}{\partial t}+u_{x} \frac{\partial u_{x}}{\partial x}+u_{r} \frac{\partial u_{x}}{\partial r}=-\frac{1}{\rho} \frac{\partial p}{\partial x}+\frac{1}{\rho} \frac{\partial \tau_{x x}^{f}}{\partial x}+\frac{1}{\rho} \frac{\partial \tau_{x r}^{f}}{\partial r}+\frac{\tau_{x r}^{f}}{\rho r}
$$

where $\tau^{f}$ represents the effective viscous stress tensor and is the sum of the real viscous stress tensor and of the Favre-averaged Reynolds-stress tensor (in RANS) or the subgrid-scale stress tensor (in LES) 42. Its components write

$$
\begin{aligned}
\tau_{x x}^{f} & =2\left(\mu_{T}+\mu\right)\left(\frac{\partial u_{x}}{\partial x}-\frac{1}{3}(\vec{\nabla} \cdot \vec{u})\right)-\frac{2}{3} \rho k \\
\tau_{x r}^{f} & =\left(\mu_{T}+\mu\right)\left(\frac{\partial u_{x}}{\partial r}+\frac{\partial u_{r}}{\partial x}\right)
\end{aligned}
$$

Equation (8)-(9) are linearized considering small amplitude perturbations and assuming that $\mu$ and $\mu_{T}$ do not depend on flow fluctuations. Density perturbations are substituted with entropy fluctuations using the linearized entropy relation $s^{\prime} / c_{p}=p^{\prime} / \gamma p_{0}-\rho^{\prime} / \rho_{0}$, with $c_{p}$ the heat capacity at constant pressure, to express the linearized mass and axial momentum equations as functions of pressure, velocity and entropy fluctuations. Temporal derivatives are then dropped considering a harmonic regime where perturbations are expressed following Eqs. (12)-(14).

$$
\begin{aligned}
u_{x}^{\prime}(x, t) & =\operatorname{Re}\left[\hat{u}(x) \mathrm{e}^{\mathrm{i} \omega t}\right] \\
p^{\prime}(x, t) & =\operatorname{Re}\left[\hat{p}(x) \mathrm{e}^{\mathrm{i} \omega t}\right] \\
\frac{s^{\prime}}{c_{p}}(x, t) & =\operatorname{Re}\left[\hat{\sigma}(x) \mathrm{e}^{\mathrm{i} \omega t}\right]
\end{aligned}
$$

To end, the momentum equation is spatially averaged over the nozzle crosssection following Eq. (7). Noticing that $\overline{p^{\prime}}=p^{\prime}$ and $\overline{u^{\prime}}=u^{\prime}$ considering the hypotheses of the model, the equations finally reduce to ordinary differential equations over velocity and pressure fluctuations as functions of the axial position and write

$\left(A \overline{\left(\frac{1}{c_{0}^{2}}\right)} \mathrm{i} \omega+\frac{\mathrm{d}}{\mathrm{d} x}\left[\overline{\left(\frac{u_{0 x}}{c_{0}^{2}}\right)}\right]\right) \hat{p}+\left[\overline{\left(\frac{u_{0 x}}{c_{0}^{2}}\right)}\right] \frac{\mathrm{d} \hat{p}}{\mathrm{~d} x}+\frac{\mathrm{d} A \bar{\rho}_{0}}{\mathrm{~d} x} \hat{u}+A \bar{\rho}_{0} \frac{\mathrm{d} \hat{u}}{\mathrm{~d} x}$ 


$$
\begin{aligned}
& =\frac{\mathrm{d}}{\mathrm{d} x}\left[A \overline{\left(\rho_{0} u_{0 x}\right) \hat{\sigma}}\right]+A \mathrm{i} \omega \overline{\rho_{0} \hat{\sigma}} \\
& \left(\mathrm{i} \omega+\overline{\left(\frac{\partial u_{0 x}}{\partial x}\right)}\right) \hat{u}+\overline{\left(u_{0 x}-\frac{4}{3 \rho_{0}} \frac{\partial\left(\mu_{T}+\mu\right)}{\partial x}\right)} \frac{\partial \hat{u}}{\partial x}-\overline{\left(\frac{4}{3 \rho_{0}}\left(\mu_{T}+\mu\right)\right)} \frac{\partial^{2} \hat{u}}{\partial x^{2}} \\
& +\overline{\left(\frac{u_{0 x}}{\gamma p_{0}} \frac{\partial u_{0 x}}{\partial x}+\frac{u_{0 r}}{\gamma p_{0}} \frac{\partial u_{0 x}}{\partial r}+\frac{2}{3 \rho_{0}} \frac{\partial}{\partial x}\left(\frac{k}{c_{0}^{2}}\right)\right)} \hat{p}+\overline{\left(\frac{1}{\rho_{0}}\left(1+\frac{2}{3} \frac{k}{c_{0}^{2}}\right)\right)} \frac{\partial \hat{p}}{\partial x} \\
& =\overline{\left(u_{0 x} \frac{\partial u_{0 x}}{\partial x}+u_{0 r} \frac{\partial u_{0 x}}{\partial r}\right) \hat{\sigma}}+\overline{\frac{2}{3 \rho_{0}} \frac{\partial\left(\rho_{0} k \hat{\sigma}\right)}{\partial x}}
\end{aligned}
$$

where $\gamma=c_{p} / c_{v}$ is the adiabatic coefficient and $c_{v}$ is the heat capacity at constant volume. From Eq. 16 the influence of viscosity on combustion noise appears to be twofold. On the left-hand-side, terms combining viscosity and pressure and velocity fluctuations represent the interaction between the turbulent, viscous mean flow and acoustic fluctuations and lead to acoustic scattering. An additional contribution is present through the interaction between acoustic perturbations and the mean flow field, which integrates viscous effects such as boundary layers. This contribution of the viscous mean flow fields is also present on the right-hand-side terms of the equation, which corresponds to the entropy noise source terms. These RHS terms also exhibit an additional term compared to the inviscid configuration, which models the interaction between turbulent kinetic energy and entropy as a supplementary noise production mechanism. As pointed out previously, this contribution of viscous terms in noise scattering and noise generation is more important with RANS flow fields than with timeaveraged LES fields because of the larger value of the eddy viscosity term and modelled turbulent kinetic energy. This is all the more true in comparison to DNS where both terms vanish.

\subsection{Numerical discretisation}

For numerical resolution of the system, the geometry is discretised with $n$ nodes along the axial direction, corresponding to $n-1$ elements at the centre 


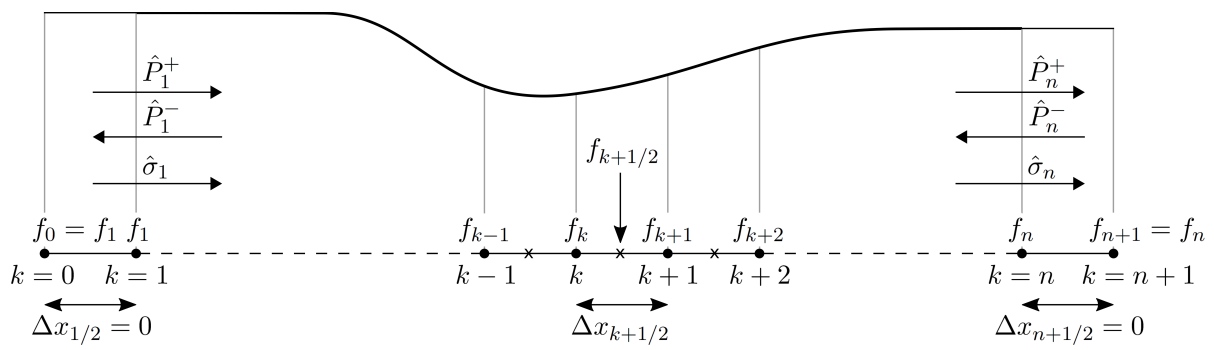

Figure 1: Numerical discretisation of the nozzle.

of which the mass and momentum equations are evaluated. The discretisation of the system is illustrated in Fig. 1. Perturbed quantities and their first- and second-order axial derivatives at the centre of each element $k+1 / 2$, bounded by nodes $k$ and $k+1$, are provided by Eqs 17$)-(19$.

$$
\begin{aligned}
f_{k+1 / 2} & =\frac{f_{k+1}+f_{k}}{2} \\
\frac{\mathrm{d}}{\mathrm{d} x} f_{k+1 / 2} & =\frac{f_{k+1}-f_{k}}{\Delta x_{k+1 / 2}} \\
\frac{\mathrm{d}^{2}}{\mathrm{~d} x^{2}} f_{k+1 / 2} & =\frac{f_{k+2}-f_{k}}{\Delta x_{k+1 / 2}\left(\Delta x_{k+1 / 2}+\Delta x_{k+3 / 2}\right)}-\frac{f_{k+1}-f_{k-1}}{\Delta x_{k+1 / 2}\left(\Delta x_{k+1 / 2}+\Delta x_{k-1 / 2}\right)}
\end{aligned}
$$

First-order derivatives correspond to a first-order Taylor expansion of the flow quantity at the centre of the nozzle, with $\Delta x_{k+1 / 2}$ the size of the element $k+$ $1 / 2$, and second-order derivatives are obtained by two successive applications of the first-order derivation operator. Second-order derivatives at the domain boundaries are evaluated with the centred operator of Eq. 19 considering $f_{0}=f_{1}$ and $\Delta x_{1 / 2}=0$ at the inlet and $f_{n+1}=f_{n}$ and $\Delta x_{n+1 / 2}=0$ at the outlet.

The discretised mass conservation and axial momentum equations in each 
element $k+1 / 2$ write

$$
\begin{aligned}
\lambda_{k+1 / 2}^{1} \hat{p}_{k}+\lambda_{k+1 / 2}^{2} \hat{u}_{k} & \\
+\lambda_{k+1 / 2}^{3} \hat{p}_{k+1}+\lambda_{k+1 / 2}^{4} \hat{u}_{k+1} & =\hat{S}_{k+1 / 2}^{C} \\
\phi_{k+1 / 2}^{0} \hat{u}_{k-1}+\phi_{k+1 / 2}^{1} \hat{p}_{k}+\phi_{k+1 / 2}^{2} \hat{u}_{k} & \\
+\phi_{k+1 / 2}^{3} \hat{p}_{k+1}+\phi_{k+1 / 2}^{4} \hat{u}_{k+1}+\phi_{k+1 / 2}^{6} \hat{u}_{k+2} & =\hat{S}_{k+1 / 2}^{M}
\end{aligned}
$$


with

$$
\begin{aligned}
& \lambda_{k+1 / 2}^{1}=\frac{1}{2}\left(A \overline{\left(\frac{1}{c_{0}^{2}}\right)} \mathrm{i} \omega+\frac{\mathrm{d}}{\mathrm{d} x}\left[A \overline{\left(\frac{u_{0 x}}{c_{0}^{2}}\right)}\right]\right)-\frac{A}{\Delta x_{k+1 / 2}} \overline{\left(\frac{u_{0 x}}{c_{0}^{2}}\right)} \\
& \lambda_{k+1 / 2}^{2}=\frac{1}{2} \frac{\mathrm{d} A \bar{\rho}_{0}}{\mathrm{~d} x}-\frac{A \bar{\rho}_{0}}{\Delta x_{k+1 / 2}} \\
& \lambda_{k+1 / 2}^{3}=\frac{1}{2}\left(A \overline{\left(\frac{1}{c_{0}^{2}}\right)} \mathrm{i} \omega+\frac{\mathrm{d}}{\mathrm{d} x}\left[A \overline{\left.\left(\frac{u_{0 x}}{c_{0}^{2}}\right)\right]}\right)+\frac{A}{\Delta x_{k+1 / 2}} \overline{\left(\frac{u_{0 x}}{c_{0}^{2}}\right)}\right. \\
& \lambda_{k+1 / 2}^{4}=\frac{1}{2} \frac{\mathrm{d} A \bar{\rho}_{0}}{\mathrm{~d} x}+\frac{A \bar{\rho}_{0}}{\Delta x_{k+1 / 2}} \\
& \phi_{k+1 / 2}^{0}=-\frac{4}{3} \frac{1}{\Delta x_{k+1 / 2}\left(\Delta x_{k+1 / 2}+\Delta x_{k-1 / 2}\right)} \overline{\left(\frac{1}{\rho_{0}}\left(\mu_{T}+\mu\right)\right)} \\
& \phi_{k+1 / 2}^{1}=\frac{1}{2}\left(\overline{\left(\frac{u_{0 x}}{\gamma p_{0}} \frac{\partial u_{0 x}}{\partial x}\right)}+\overline{\left(\frac{u_{0 r}}{\gamma p_{0}} \frac{\partial u_{0 x}}{\partial r}\right)}+\frac{2}{3} \overline{\left(\frac{1}{\rho_{0}} \frac{\partial}{\partial x}\left[\frac{k}{c_{0}^{2}}\right]\right)}\right) \\
& -\frac{1}{\Delta x_{k+1 / 2}} \overline{\left(\frac{1}{\rho_{0}}\left(1+\frac{2}{3} \frac{k}{c_{0}^{2}}\right)\right)} \\
& \phi_{k+1 / 2}^{2}=\frac{1}{2}\left(\mathrm{i} \omega+\overline{\left(\frac{\partial u_{0 x}}{\partial x}\right)}\right)-\frac{1}{\Delta x_{k+1 / 2}}\left(\bar{u}_{0 x}-\frac{4}{3} \overline{\left(\frac{1}{\rho_{0}} \frac{\partial\left(\mu_{T}+\mu\right)}{\partial x}\right)}\right) \\
& +\frac{4}{3} \frac{1}{\Delta x_{k+1 / 2}\left(\Delta x_{k+1 / 2}+\Delta x_{k+3 / 2}\right)} \overline{\left(\frac{1}{\rho_{0}}\left(\mu_{T}+\mu\right)\right)} \\
& \phi_{k+1 / 2}^{3}=\frac{1}{2}\left(\overline{\left(\frac{u_{0 x}}{\gamma p_{0}} \frac{\partial u_{0 x}}{\partial x}\right)}+\overline{\left(\frac{u_{0 r}}{\gamma p_{0}} \frac{\partial u_{0 x}}{\partial r}\right)}+\frac{2}{3} \overline{\left(\frac{1}{\rho_{0}} \frac{\partial}{\partial x}\left[\frac{k}{c_{0}^{2}}\right]\right)}\right) \\
& +\frac{1}{\Delta x_{k+1 / 2}} \overline{\left(\frac{1}{\rho_{0}}\left(1+\frac{2}{3} \frac{k}{c_{0}^{2}}\right)\right)} \\
& \phi_{k+1 / 2}^{4}=\frac{1}{2}\left(\mathrm{i} \omega+\overline{\left(\frac{\partial u_{0 x}}{\partial x}\right)}\right)+\frac{1}{\Delta x_{k+1 / 2}}\left(\bar{u}_{0 x}-\frac{4}{3} \overline{\left(\frac{1}{\rho_{0}} \frac{\partial\left(\mu_{T}+\mu\right)}{\partial x}\right)}\right) \\
& +\frac{4}{3} \frac{1}{\Delta x_{k+1 / 2}\left(\Delta x_{k+1 / 2}+\Delta x_{k-1 / 2}\right)} \overline{\left(\frac{1}{\rho_{0}}\left(\mu_{T}+\mu\right)\right)} \\
& \phi_{k+1 / 2}^{6}=-\frac{4}{3} \frac{1}{\Delta x_{k+1 / 2}\left(\Delta x_{k+1 / 2}+\Delta x_{k+3 / 2}\right)} \overline{\left(\frac{1}{\rho_{0}}\left(\mu_{T}+\mu\right)\right)} \\
& \hat{S}_{k+1 / 2}^{C}=\frac{\mathrm{d}}{\mathrm{d} x}\left[A \overline{\left(\rho_{0} u_{0 x} \hat{\sigma}\right)}\right]+A \mathrm{i} \omega \overline{\left(\rho_{0} \hat{\sigma}\right)} \\
& \hat{S}_{k+1 / 2}^{M}=\overline{\left(\left[u_{0 x} \frac{\partial u_{0 x}}{\partial x}+u_{0 r} \frac{\partial u_{0 x}}{\partial r}\right] \hat{\sigma}\right)}+\frac{2}{3} \overline{\left(\frac{1}{\rho_{0}} \frac{\partial\left(\rho_{0} k \hat{\sigma}\right)}{\partial x}\right)}
\end{aligned}
$$

In these expressions the flow variables are evaluated inside the element $k+1 / 2$, which is not explicitly written for the sake of clarity. The second term of $\hat{S}_{k+1 / 2}^{M}$ 
includes the axial derivative of a product between the mean flow variables and the entropy fluctuation. Depending on how the entropy fluctuation is computed this derivative may not be easily evaluable (see for instance the method proposed in 3.4 . This expression can be approximated by Eq. (34), whose derivation is detailed in Appendix B, and which is valid for small mean flow angles with respect to the axial direction.

$$
\hat{S}_{k+1 / 2}^{M} \simeq \overline{\left(\left[u_{0 x} \frac{\partial u_{0 x}}{\partial x}+u_{0 r} \frac{\partial u_{0 x}}{\partial r}\right] \hat{\sigma}\right)}+\frac{2}{3} \overline{\left(\left[-\frac{\mathrm{i} \omega k}{u_{0}} \frac{\partial l}{\partial x}+\frac{1}{\rho_{0}} \frac{\partial\left(\rho_{0} k\right)}{\partial x}\right] \hat{\sigma}\right)}
$$

In this equation, $l$ stands for the curvilinear abscissa along the streamlines.

\subsection{Boundary conditions}

The evaluation of mass and momentum equations inside the $n-1$ axial elements discretising the geometry provides $2 n-2$ equations, whereas $2 n$ unknowns corresponding to $\hat{u}$ and $\hat{p}$ at the $n$ nodes need to be determined. The last two equations are provided by the inlet and outlet boundary conditions. For the sake of simplicity, it is considered in the present study that there is no acoustic reflection on the boundaries so that the acoustic waves entering the domain correspond to the imposed acoustic forcings. Using the expressions of the Riemann invariants derived in $\$ 2$, Eq. (6), this translates in the numerical domain to Eqs $35-36$, where $P_{1, f}^{+}$and $P_{n, f}^{-}$correspond respectively to the upstream and downstream acoustic forcings imposed by the user. In these equations, the subscripts $(\cdot)_{1}$ and $(\cdot)_{n}$ indicate that the flow quantities are evaluated at nodes 1 and $n$.

$$
\begin{aligned}
& \hat{P}_{1}^{+}=\hat{P}_{1, f}^{+}=\frac{1}{2}\left(\frac{\hat{p}}{\gamma \bar{p}_{0}}+\frac{\overline{\rho_{0} c_{0}}}{\gamma \bar{p}_{0}} \hat{u}\right)_{1} \\
& \hat{P}_{n}^{-}=\hat{P}_{n, f}^{-}=\frac{1}{2}\left(\frac{\hat{p}}{\gamma \bar{p}_{0}}-\frac{\overline{\rho_{0} c_{0}}}{\gamma \bar{p}_{0}} \hat{u}\right)_{n}
\end{aligned}
$$

\subsection{Modelling of the entropy fluctuations}

The last part of the modelling concerns the evaluation of the entropy fluctuations inside the nozzle. For the subsonic flows considered here, entropy is not affected by the acoustics and can be computed prior to the resolution of Eqs. 
(15)-16. This is however not always the case, e.g. when entropy fluctuations

are modified through the shock and need to be computed jointly with acoustics, see for instance Huet et al. [26].

Several possibilities can be considered for the computation of the entropy fluctuations, depending on the level of modelling desired. The numerical resolution of the convection equation for entropy may be considered when effects such as thermal diffusion or turbulent mixing need to be captured. In this section, a simplified approach corresponding to the pure convection of entropy is used. This method proved to reproduce shear dispersion of entropy with an excellent agreement when Euler equations are considered [25] and it is reasonable to assume thermal diffusion is negligible when pressurized combustors are considered [36, 37.

The method is based on streamlines, constructed from the mean flow field. These streamlines are used to define streamtubes, in which the flow is assumed to be radially uniform. This hypothesis is verified if a sufficient number of streamtubes is considered, i.e. if their height is sufficiently small. Entropy fluctuations being purely convected by the flow, they remain confined in the same streamtube throughout the nozzle. Under these assumptions, entropy can be expressed analytically at every position inside the nozzle for each streamtube and writes in the harmonic regime [25, 26]

$$
\hat{\sigma}(l)=\hat{\sigma}(l=0) \exp \left(-\mathrm{i} \omega \int_{0}^{l} \frac{\mathrm{d} \zeta}{u_{0}(\zeta)}\right)
$$

with $l$ the curvilinear abscissa along the streamtube, $\hat{\sigma}(l=0)$ the complex amplitude of the entropy fluctuation at the domain inlet and $u_{0}=\sqrt{u_{0 x}^{2}+u_{0 r}^{2}}$ the mean flow velocity.

\subsection{Equations in matrix form}

Combining Eqs (20)-(21) and Eqs. (35)-(36), the linear system to solve writes in the matrix form as Eq. (38), that can be inverted numerically for each frequency. There is a total of $2 n+4$ unknowns in this equation because 
ghost nodes and associated relations have been added at nozzle boundaries to

allow the use of the centred second-order derivative operator for all geometry elements, as discussed in 33.2 . Transfer functions are then reconstructed from the computed pressure and velocity perturbations at both extremities using Eq. (6). This numerical resolution is performed in the present study with the in-

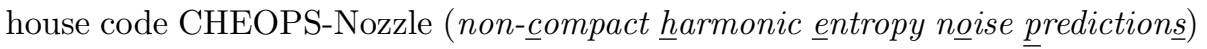
275 [25, 26].

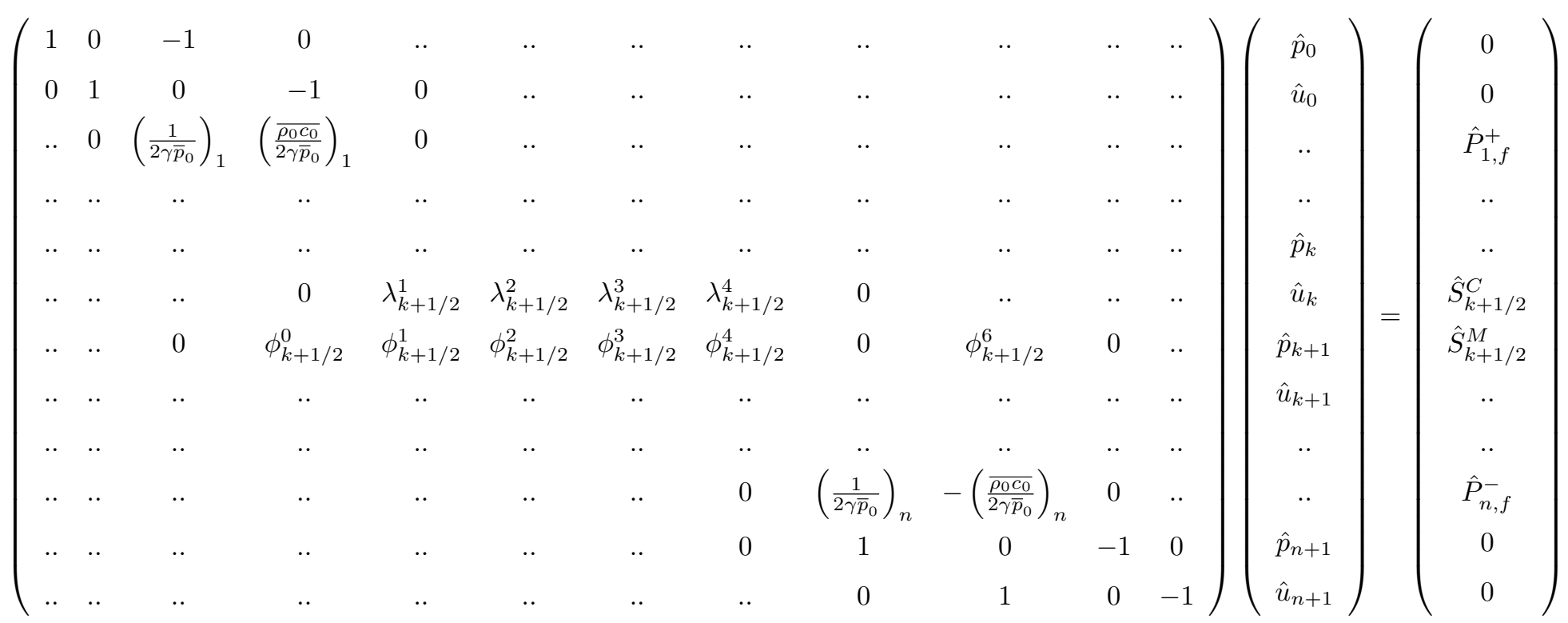

\section{Numerical simulations}

\subsection{Geometry and operating conditions}

The geometry considered is the converging-diverging DISCERN nozzle designed in the framework of the ANR DISCERN project to investigate indirect

combustion noise. It was previously used for the validation of the inviscid version of the 2D model [25, 26] and is illustrated below in Fig 2, The nozzle is $185 \mathrm{~mm}$ long (100 $\mathrm{mm}$ for the convergent and $85 \mathrm{~mm}$ for the divergent) and its radius varies from $29.5 \mathrm{~mm}$ at the inlet to $6.943 \mathrm{~mm}$ at the outlet, with a 
minimum of $5.5 \mathrm{~mm}$ at the throat. It is complemented by $100 \mathrm{~mm}$ long ducts of constant section at both extremities to achieve a separation of the acoustic waves in the numerical simulations, as detailed in $\$ 4.4$.

The operating point considered is very similar to the one used by Emmanuelli et al. [25], with the exception of the back pressure set to 30 bar. This high pressure value is representative of the pressure inside typical combustion chamber. Inlet temperature and Mach number are $1300 \mathrm{~K}$ and 0.0179. For the Euler configuration, it leads to a Mach number of 0.658 at nozzle throat and 0.344 at nozzle exit. To end, the fluid considered is air with constant heat capacities and $\gamma=1.315$.

\subsection{Methodology}

Numerical simulations are used to determine the noise scattered (acoustic transfer functions) or generated by the acceleration of entropy spots (thermoacoustic transfer functions) through the nozzle with both inviscid and viscous flow fields. Inviscid flow simulations correspond to the resolution of the Euler equations whereas $k-\omega$ SST URANS equations are considered when viscosity is taken into account. Simulations are 2D axisymmetric and are performed with the unstructured flow solver CEDRE from ONERA 44. The numerical grid used is the same for Euler and URANS simulations and is identical to that previously used by Emmanuelli et al. [25]. Nozzle walls are discretised with 30 layers of quadrilateral cells and ensure a flow resolution with $y^{+}=1$ at the walls in RANS, whereas the rest of the domain is filled with triangles sufficiently small to ensure that mean flow gradients are correctly captured and numerical dissipation of acoustic and entropy waves is negligible for the frequencies considered. The mesh is composed of 1,800,000 elements. Spatial derivatives are evaluated with a second-order space scheme and the implicit second-order Runge-Kutta scheme is used in time. The time step is chosen to ensure a CFL criterion below 1 in most of the numerical domain.

Three simulations are performed for both inviscid and viscous configurations, corresponding to entropy and acoustic forcings from the upstream limit and 
acoustic forcing from the downstream limit. Acoustic and entropy waves are

but it is reasonable to consider they remain valid for nozzle flows. In addition, an accurate evaluation of the turbulent thermal diffusion would require an LES with turbulence representative of the exhaust of a combustion chamber, including flame-induced and wall-cooling turbulence, which is out of the scope of the hence their associated noise sources, are similar in both inviscid and viscous simulations. Having different entropy perturbation profiles between Euler and URANS simulations would complicate the evaluation of the viscosity effect on the thermo-acoustic transfer functions of the nozzle. 
When the inviscid equations are solved the flow is uniform in the upstream and downstream ducts; therefore uniform static temperature and velocity are imposed on the inlet boundary and uniform static pressure is imposed on the outlet boundary. For the viscous simulation, on the contrary, inlet and outlet profiles need to reproduce the boundary layer that develops on the duct wall. In the present simulation, the boundary layer velocity profile is defined by Eqs. (39)-(40), issued from Bogey et al. [48] and corresponding to a polynomial approximation of a laminar boundary layer with a Blasius profile

$$
\begin{aligned}
& u_{x}\left(r_{w}\right)=U_{0} \frac{r_{w}}{\delta_{B}}\left[2-2\left(\frac{r_{w}}{\delta_{B}}\right)^{2}+\left(\frac{r_{w}}{\delta_{B}}\right)^{3}\right] \text { if } r_{w}<\delta_{B} \\
& u_{x}\left(r_{w}\right)=U_{0} \text { if } r_{w} \geq \delta_{B}
\end{aligned}
$$

where $U_{0}$ is the flow velocity outside the boundary layer (same velocity as in the Euler simulation), $r_{w}$ the distance to the wall and $\delta_{B}$ the boundary layer thickness. Following the work of Bogey et al. [48, the boundary layer thickness is set to $6.4 \%$ of the duct diameter, $\delta_{B}=3.8 \mathrm{~mm}$. The profile of the turbulent kinetic energy is deduced from the velocity profile considering an isotropic homogeneous turbulence with the turbulence level being $5 \%$ of the mean velocity

$$
k=3 / 2 \times\left(0.05 \times u_{x}\right)^{2}
$$

and $\omega$ is obtained from $k$ using the relation

$$
\omega=\frac{\sqrt{k}}{l}
$$

where $l$ is the turbulent length scale, $l=0.038 D$ with $D$ the duct diameter. To end, the temperature variation is evaluated using the adiabatic relation:

$$
T_{w}=T_{0}\left(1+\frac{\gamma-1}{2} M_{0}^{2}\right)
$$

with $T_{w}$ the temperature at the wall and $T_{0}$ and $M_{0}$ the temperature and Mach number outside of the boundary layer. Temperature variations below $0.1 \mathrm{~K}$ are 


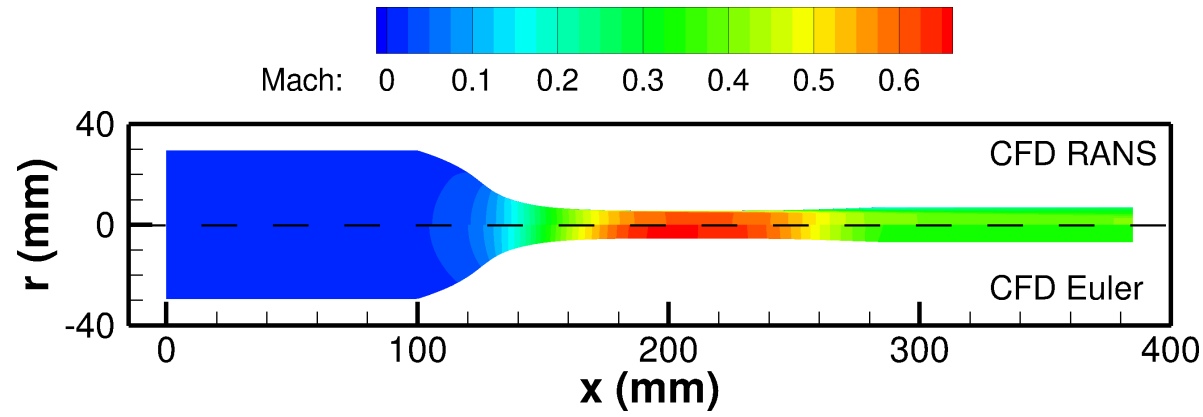

(a)

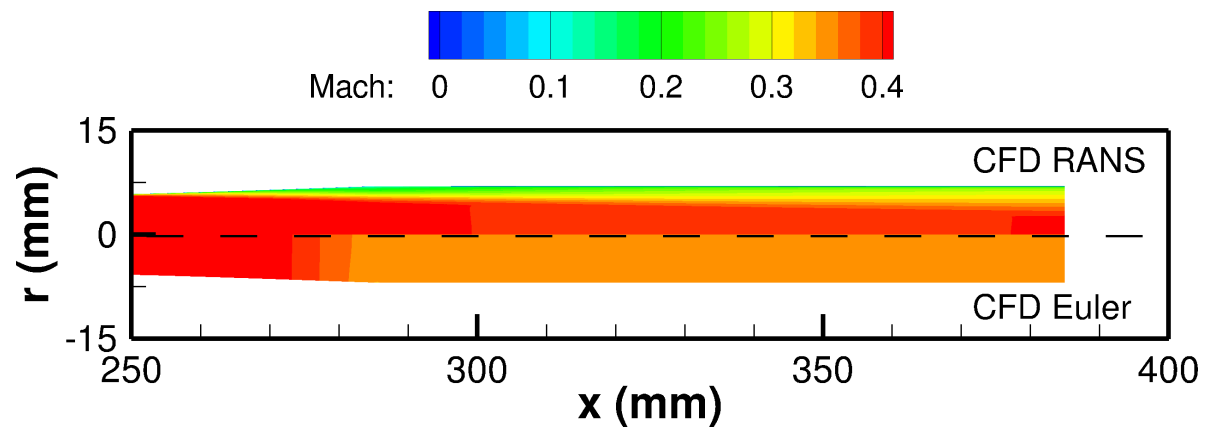

(b)

Figure 2: Mean Mach number evolution along the nozzle for RANS (top) and Euler (bottom) simulations. (a) global view, (b) zoom on the downstream duct

found (in comparison to the mean temperature of $1300 \mathrm{~K}$ ), so that a uniform temperature profile is imposed at the inlet.

At the downstream end, the boundary layer develops freely and is not known a priori, therefore the pressure profile cannot be imposed. Instead, the sectionaveraged pressure is imposed on the exit surface, so that pressure can locally vary along the radial direction to reproduce the boundary layer profile.

The use of these boundary conditions leads to slightly different mass flow rates between the two simulations. On the one hand, inlet pressure and hence density is expected to be higher for the RANS simulation because of the pressure loss along the nozzle, which tends to increase its mass flow rate. On the other hand, the mass flow rate is decreased in the viscous simulation due to the 
presence of the boundary layer. The combination of both effects leads to a decreased mass flow rate of $4.9 \%$ for the RANS compared to the Euler simulation $(0.2975 \mathrm{~kg} / \mathrm{s}$ for Euler and $0.2829 \mathrm{~kg} / \mathrm{s}$ for RANS $)$

The evolution of the Mach number along the nozzle is illustrated in Fig. 2 for both simulations. The Mach number barely depends on the radial position for the Euler simulation. A similar observation is made for the RANS upstream of the throat, where the boundary layer is very thin. It nevertheless broadens quickly in the divergent part and is quite thick in the downstream duct, leading to large mean flow variations along the radius as illustrated in Fig. 2 (b). A consequence of this thick boundary layer is the much larger Mach number on the nozzle axis in RANS in the downstream region, where it rises to 0.40 in comparison to 0.34 in the Euler simulation.

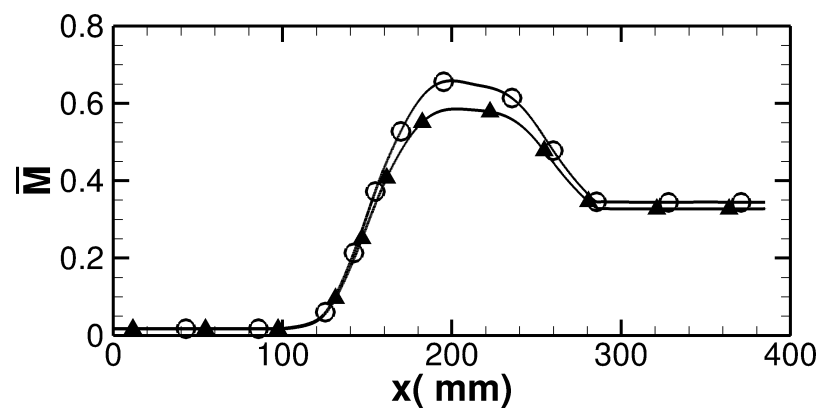

(a)

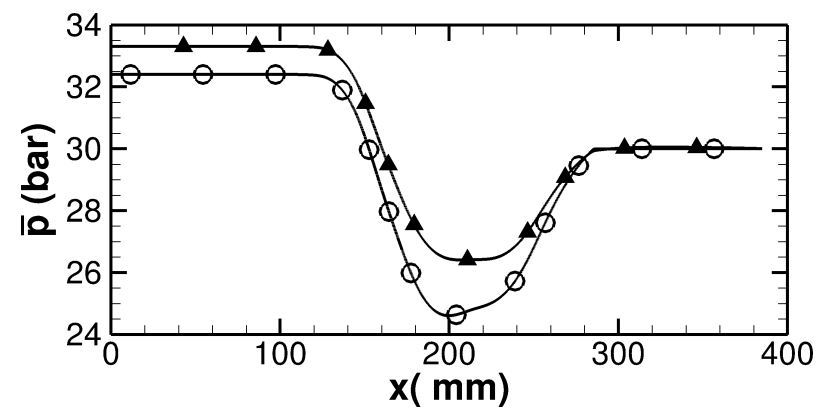

(b)

Figure 3: Axial evolution of section-averaged (a) Mach number and (b) pressure. $\longrightarrow$ CFD Euler; - $\triangle$ CFD RANS. 
More detailed comparisons of the Mach number evolution along the nozzle and at the exit (0.327). The pressure loss due to viscosity is illustrated in Fig. 3 (b) and leads to a pressure variation of 0.9 bar between the two simulations at the nozzle inlet.

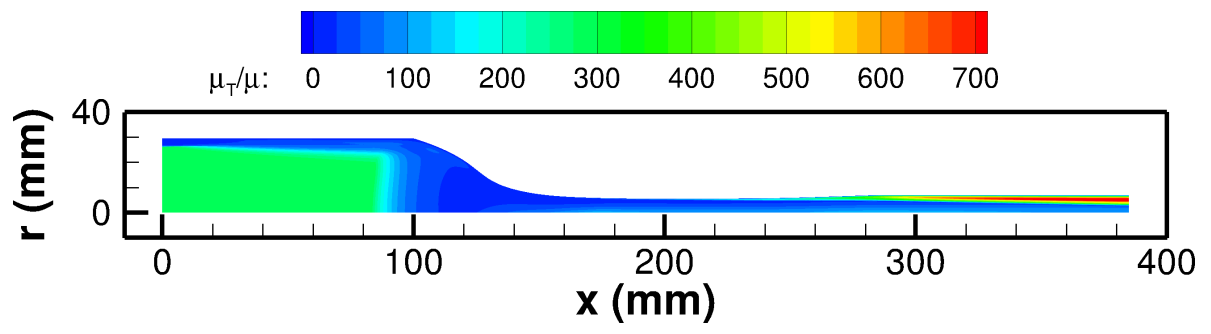

are provided in Fig. 3, which reproduces the section-averaged Mach number and pressure evolution as a function of the axial position. As indicated previously, the Euler flow is accelerated from Mach 0.0179 at the inlet to 0.658 at the throat, then decelerated towards 0.344 at the exit. Due to the boundary layer, the section-averaged Mach number is slightly lower in the upstream duct (0.0165) for the RANS, which explains the lower values also observed at the throat (0.585)

Figure 4: Evolution of the viscosity ratio $\mu_{T} / \mu$ along the nozzle for RANS simulation.

\section{The viscosity ratio $\mu_{T} / \mu$ obtained with the RANS simulation is shown in} Fig.4. As mentioned during the derivation of the model, large values of this ratio correspond to zones where acoustic perturbations are scattered by modelled turbulence. Inside the nozzle, turbulent viscosity remains limited and reaches a maximum value of about 100 times the physical viscosity, with the exception of the boundary layer at the very end of the nozzle where it rises very locally to 395 400. Larger values are observed in the core of the upstream duct $\left(\mu_{T} / \mu \sim 300\right)$ and in the boundary layer of the downstream duct $\left(\mu_{T} / \mu \leq 700\right)$. If present, the scattering of acoustic waves by modelled turbulence is therefore expected to occur essentially in the upstream and downstream ducts.

Finally, the evolution of the modelled turbulent kinetic energy and its axial gradient are reproduced in Fig. 5. These two quantities are of interest because 


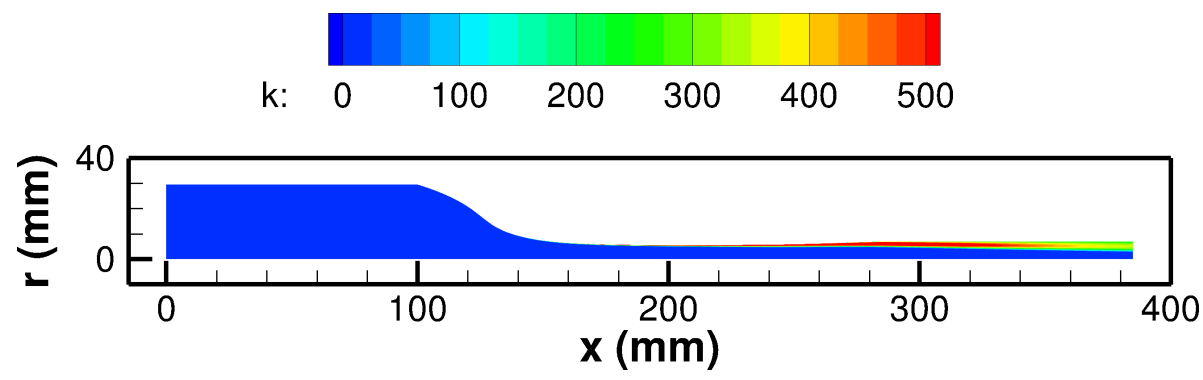

(a)

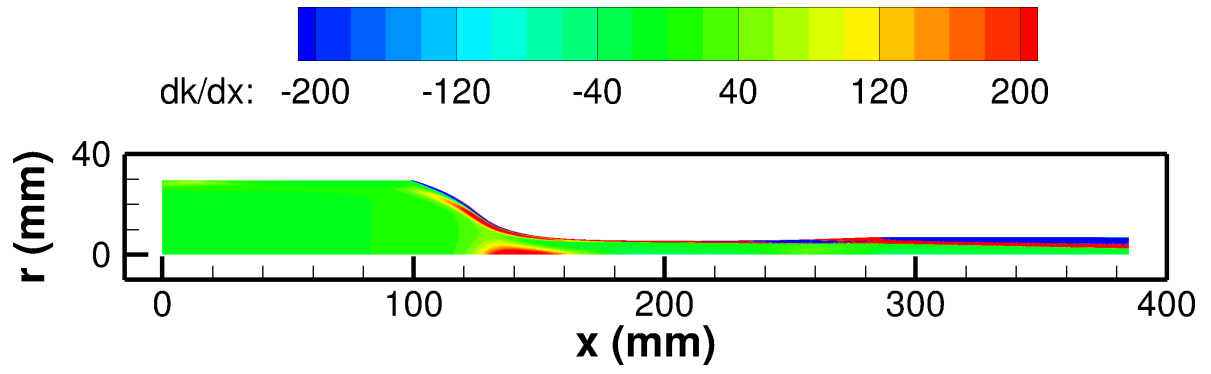

(b)

Figure 5: Evolution of (a) the turbulent kinetic energy $k$ and (b) its axial gradient inside the nozzle for RANS simulation.

they drive the intensity of the viscous entropy noise source term, see Eq. (34). Turbulent kinetic energy is low in most of the domain and its largest value is observed in the boundary layer at the end of the divergent where its axial derivative is also high. Additionally, the axial gradient presents large levels near the wall and near the axis of the convergent part. Entropy noise source terms associated with viscous effects are thus expected to be observed in these two regions of the flow.

\subsection{Numerical transfer functions}

Once the mean flows are computed, acoustic or entropic fluctuations are injected through the boundaries. Noise is scattered or generated inside the nozzle and the resulting waves are used to construct the numerical transfer functions of the nozzle. Three different forcings are considered: acoustic from 
the inlet, acoustic from the outlet and entropic from the inlet. These forcings are achieved by superimposing perturbations to the imposed mean flow quantities. It is chosen here to force the nozzle with plane waves. The shear layer in RANS nevertheless produces a strong shearing of the entropy fluctuation, which is quickly dissipated numerically close to the wall. To allow fair comparisons between RANS and Euler simulations, the inlet entropy fluctuation is set to 0 near the nozzle wall $(r>0.0265 \mathrm{~m})$ for all simulations. It is worth noting such attenuation of the entropy fluctuations may be expected experimentally in the boundary layers through shear dispersion and turbulent mixing.

Construction of the transfer functions requires the evaluation of entropy and acoustic waves upstream and downstream of the nozzle. This wave separation process is carried out in the ducts. The planar inlet entropy wave is obtained from its definition, $\sigma=s^{\prime} / c_{p}$, and acoustic waves are computed using the Riemann invariants, see Eq. (6). This definition of the acoustic waves is theoretically valid when all fluctuations are of acoustic origin only, which is not the case here. Indeed, vorticity fluctuations may be generated through the nozzle 24] and produce additional velocity perturbations in the downstream duct. Such perturbations are removed through a characteristic filtering of the acoustic waves in each duct, performed along 9 evenly located stations [49. In addition, despite the use of non-reflective boundary conditions, small spurious acoustic reflections may occur on the domain boundaries. To get rid of these reflections, non-reflective post-processing is performed through the writing of a linear system where the unknowns are the nozzle transfer functions and the parameters are the upstream and downstream computed waves. Details of this post-processing method are given in Emmanuelli et al. 25]. This postprocessing proved to be successful in previous studies performed at ONERA [45]. Simulated transfer functions are illustrated in Figs. 6 6 7 and Fig. 12 and ${ }_{440}$ are discussed in the next section, along with the model predictions. 


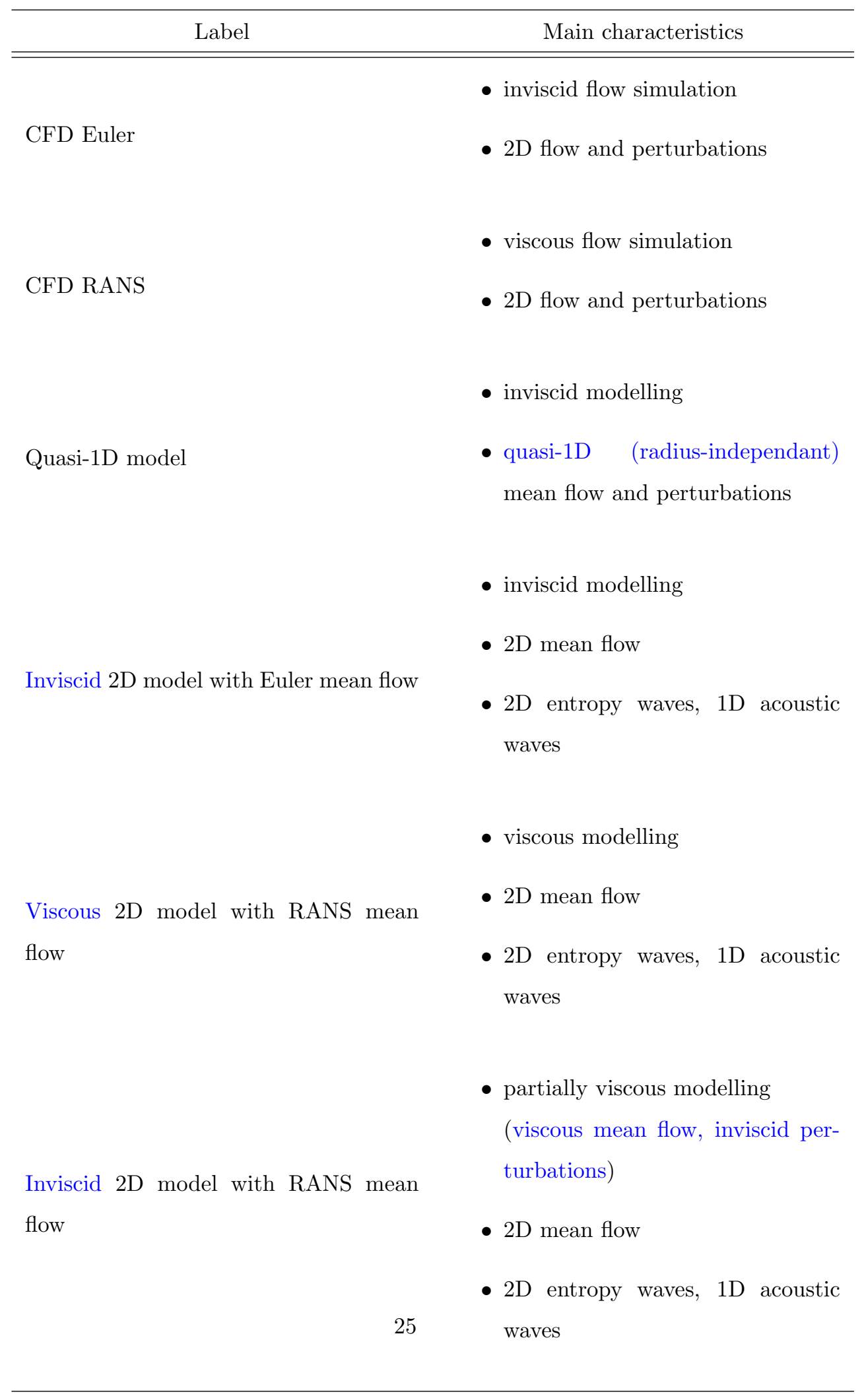

Table 1: Nomenclature and main characteristics of the different methods used to evaluate the transfer functions. 


\section{Comparisons between simulated and modelled transfer functions}

The influence of viscosity on acoustic and thermo-acoustic nozzle transfer functions is evaluated in this section through numerical simulations and analytical modelling. The different models used in the rest of the paper are summarised

in Table 1 where their main characteristics are recalled. These characteristics are described in more detail hereafter.

The quasi-1D model refers to the quasi-1D assumption, where the flow is inviscid and all variables are uniform along the radial direction (spatial dependance in the $x$ direction only) $18,20,22$. With these assumptions, acoustic and entropy waves are planar and vorticity perturbations are nil. For the sake of simplicity, in the present study the quasi-1D transfer functions are computed with the 2D model. The quasi-1D mean flow is provided analytically through mass conservation and the 1D character of the entropy wave is ensured by the use of a unique streamtube that extends radially from nozzle axis to nozzle wall.

The viscous 2D model is the model detailed in Section 3 whereas the inviscid 2D model corresponds to the inviscid formulation of the model, obtained by zeroing all viscous terms $\left(\mu=\mu_{T}=0 \mathrm{~kg} / \mathrm{m} / \mathrm{s}\right.$ and $\left.k=0 \mathrm{~m}^{2} / \mathrm{s}^{2}\right)$ in Eqs 22 (33). The inviscid 2D model is identical to the one presented in Emmanuelli et al. 25. In the viscous $2 \mathrm{D}$ model with RANS mean flow, viscosity is accounted for both in the mean flow evaluation and in the propagation of the perturbations, whereas all viscous effects are neglected using the inviscid 2D model with Euler mean flow. Midway between these two models, the inviscid 2D model with RANS mean flow accounts for viscosity only through the mean flow for the convection of entropy and noise-associated sources, as well as for the scattering of the acoustic waves.

With the 2D models, Both mean flow and entropy fluctuations depend on the radial position and are hence $2 \mathrm{D}$, whereas acoustic waves are assumed to be 1D. In addition, it is worth recalling vorticity is neglected in these models. To end with 2D modelling, a convergence study was conducted and demonstrated that the results were stable if at least 100 streamtubes were considered. This 
value is used throughout the rest of the document.

\subsection{Acoustic forcing}

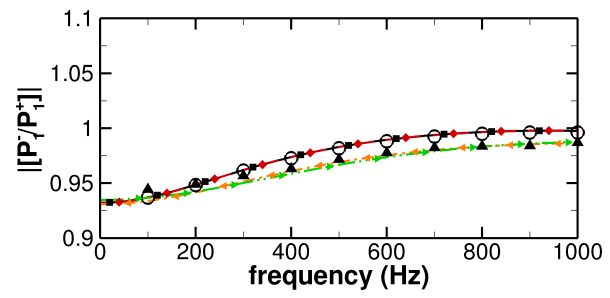

(a)

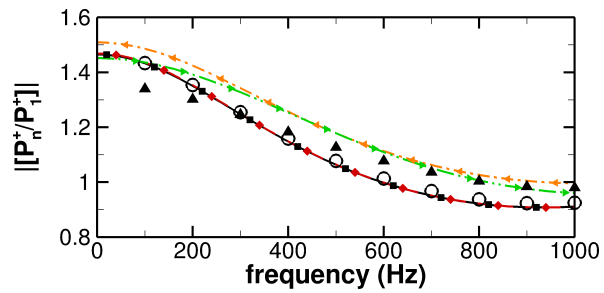

(c)

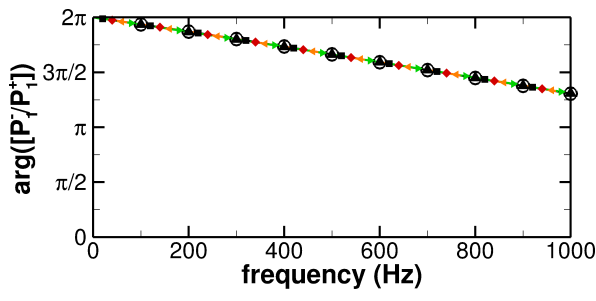

(b)

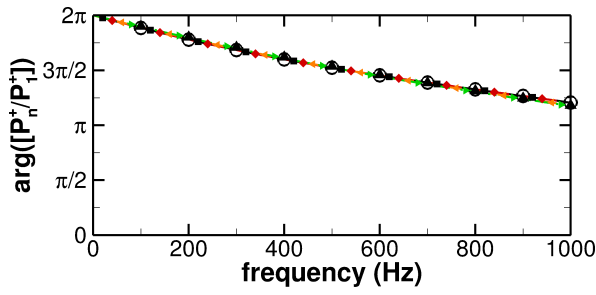

(d)

Figure 6: Computed and modelled nozzle transfer functions with upstream acoustic forcing $P_{1}^{+}$. (a) upstream wave, amplitude, (b) upstream wave, phase, (c) downstream wave, amplitude, (d) downstream wave, phase. o CFD Euler; $\boldsymbol{\Delta}$ CFD RANS; - - quasi-1D model; - inviscid 2D model with Euler mean flow; $-\cdots$ viscous 2D model with RANS mean flow; 4 inviscid 2D model with RANS mean flow.

The transfer functions simulated with CFD Euler and CFD RANS approaches are reproduced in Fig. 6 for the upstream acoustic forcing $P_{1}^{+}$. Several modelled transfer functions are also reproduced in the figure. Modelled transfer functions with Euler flow present excellent agreement with the associated simulation. They correspond to the results of Emmanuelli et al. [25] and their agreement will not be discussed further. Simulated transfer functions are slightly modified when viscosity is considered. Specifically, the reflected acoustic wave $\left[P_{1}^{-} / P_{1}^{+}\right]$is slightly lowered in the medium to high frequency range whereas it is weakly increased at low frequencies. Similar modifications have been observed numerically by Becerril for the EWG nozzle using 3D LES and Euler simulations 
34. This medium- and high-frequency noise decrease is well captured by the model, which nevertheless fails to reproduce the slight low-frequency increase. The solutions obtained with and without considering viscous terms in the model display almost identical results, which indicates that the modification of the reflection coefficient is driven by the mean flow field, in particular the presence of the boundary layer, and not by viscous effects on the perturbations. Observations are very similar for the transmitted wave $\left[P_{n}^{+} / P_{1}^{+}\right]$. Numerically, the transmission coefficient is increased for high frequencies and decreased for low frequencies with viscosity. The high-frequency increase is correctly captured by the model but not the low-frequency reduction. Taking into account the viscous terms in the model -viscous 2D model with RANS mean flow- slightly improves the comparison with the numerical simulation in regard to the inviscid 2D model with RANS mean flow fields, but differences are very low. Concerning the phase of the transfer functions, an excellent agreement is observed between both simulations and all the modelled reflection and transmission coefficients, which indicates that the presence of the boundary layer does not affect the propagation velocity of the acoustic waves.

Next, the transfer functions corresponding to the downstream acoustic forcing $P_{n}^{-}$are reproduced in Fig. 7. As previously observed with the upstream acoustic forcing, computed (CFD) transmission and reflection coefficients are very similar when considering viscosity or not. In the present case, for both $\left[P_{1}^{-} / P_{n}^{-}\right]$and $\left[P_{n}^{+} / P_{n}^{-}\right]$this leads to similar transfer functions at high-frequency 505 and to amplitude reductions in the low-frequency range when viscosity is included. This is again globally in agreement with the findings of Becerril 34. The noise reduction is qualitatively reproduced by the low-order model when viscous terms are included, despite being underestimated. With the quasi-1D model all the fluctuations are of acoustic origin and no dissipation occurs, so that all the incident acoustic energy is either transmitted or reflected by the nozzle [29]. The reduced amplitudes of the simulated transfer functions at low-frequencies with CFD RANS indicate that part of the acoustic energy is dissipated by viscosity, a behaviour captured -at least qualitatively- by the 


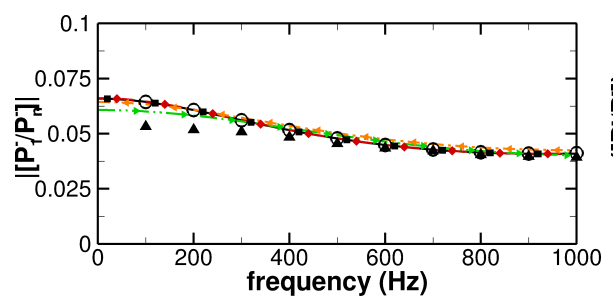

(a)

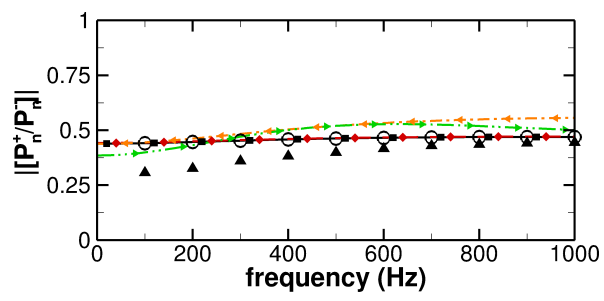

(c)

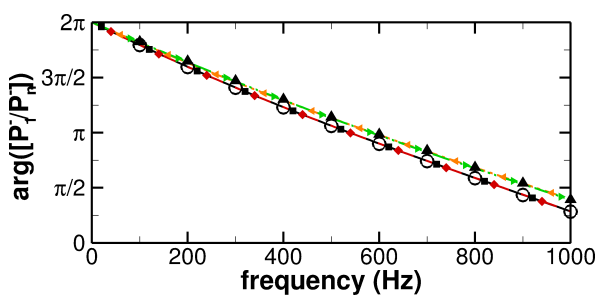

(b)

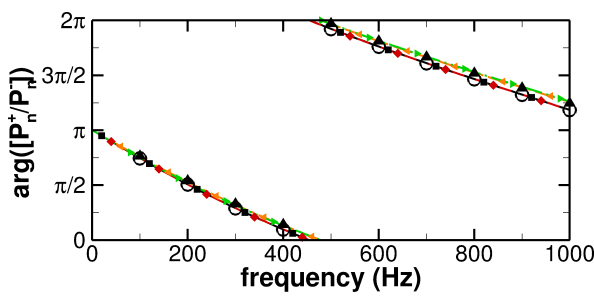

(d)

Figure 7: Computed and modelled nozzle transfer functions with downstream acoustic forcing $P_{n}^{-}$. (a) upstream wave, amplitude, (b) upstream wave, phase, (c) downstream wave, amplitude, (d) downstream wave, phase. See Fig. 6 for legend.

model. Concerning the medium- and high-frequency parts of the transfer functions, they are correctly reproduced for the transmission coefficient but slightly overpredicted for the reflection one. The agreement is nevertheless globally satisfactory. Differences are observed in the phase of the simulated transfer functions between CFD Euler and RANS. This indicates small variations in acoustic propagation velocity in these cases, but the discrepancies remain small. In addition, these phase variations are correctly captured by the model.

To understand the origin of the slight discrepancies between the numerical simulations and the model with a viscous mean flow field, Fig. 8 reproduces the pressure fluctuation along the downstream part of the nozzle for a harmonic downstream acoustic forcing $P_{n}^{-}$at $400 \mathrm{~Hz}$. For the inviscid configuration, the pressure fluctuation does not vary with the radial position, as assumed in the model. Planar pressure fluctuations are also observed in the RANS simulations near the nozzle throat where the boundary layer is thin, but radial variations 


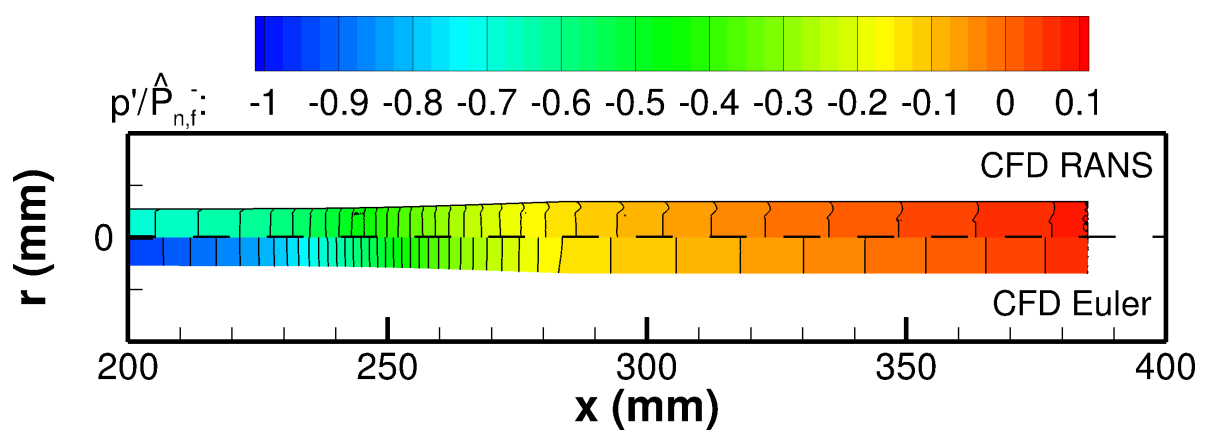

Figure 8: Simulated pressure fluctuation inside the nozzle with a downstream harmonic acoustic forcing at $400 \mathrm{~Hz}$. Isolines are added to help comparison.

become more and more visible towards the end of the nozzle as the boundary layer thickens. These radial fluctuations cannot be captured by the model by definition and may explain the differences observed with the simulations. This distortion however remains sufficiently low for the separation of acoustic waves to remain valid under the planar acoustic wave assumption. In addition, it is worth noting it has been verified that vorticity remains negligible in RANS simulations and does not contribute to noise scattering.

To summarize the results obtained so far, numerical simulations proved viscosity does not strongly modify the scattering of acoustic waves through the nozzle. The key element to capture viscous effects with the model is to take into account the boundary layer of the mean flow field. Eddy viscosity only adds a minor contribution in modelling noise scattering, but its inclusion in the model reduces the discrepancies between the numerical and predicted transfer functions. In the last part of the paper, thermo-acoustic transfer functions of the nozzle corresponding to the noise generated by the acceleration of entropy fluctuations are investigated. These transfer functions combine the generation of entropy noise inside the nozzle and its scattering by the mean flow. 


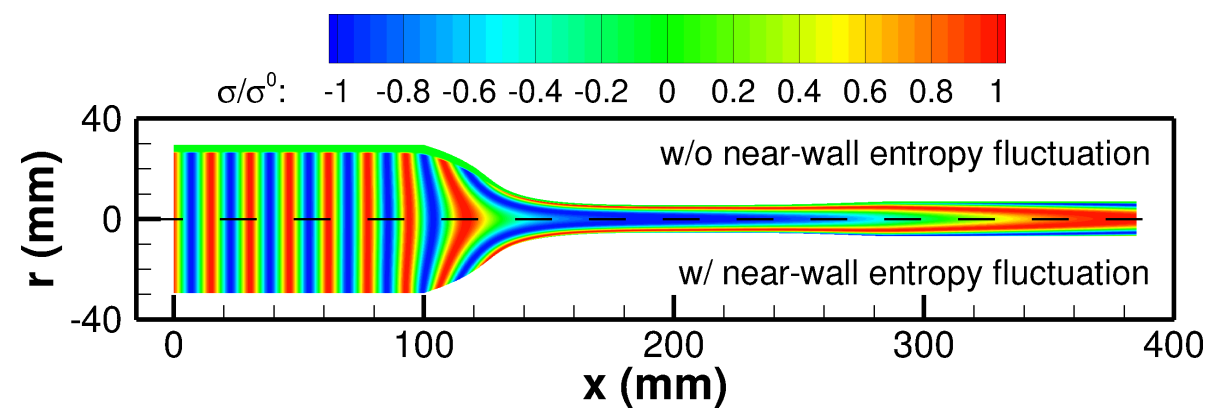

Figure 9: 2D modelled entropy fluctuation at $800 \mathrm{~Hz}$ with Euler mean flow without (top) and with (bottom) entropy fluctuation near the wall.

\subsection{Entropy forcing}

\subsubsection{Influence of the shape of the entropy wave on entropy noise generation}

In a previous study, Emmanuelli et al. 25] demonstrated the capacity of the inviscid 2D model with Euler mean flow to predict the entropy transfer functions of the nozzle with an excellent agreement for planar inlet entropy waves. In the present study, nevertheless, such planar waves are not considered because the near-wall entropy fluctuations are dissipated in the boundary layer in the viscous simulation (CFD RANS), which would make comparisons between CFD RANS and viscous 2D model with RANS mean flow unreliable. Prior to discussing viscous effects on entropy noise generation, the influence of the shape of the entropy wave on noise generation is hence discussed in this section for inviscid flows. The variation in the shape of the entropy wave corresponds to the absence of entropy fluctuations near the wall, as illustrated in Fig. 9 .

Simulated and modelled transfer functions are reproduced in Fig. 10. It is recalled that the transfer functions correspond to the generated acoustic waves $P_{1}^{-}$and $P_{n}^{+}$(one dimensional waves in the upstream and downstream ducts) divided by the section-averaged entropy fluctuations $\bar{\sigma}_{1}$. In the following, the overbar is dropped in the definition of the transfer functions for the sake of simplicity. First, it is observed in Fig. 10 that all simulated and modelled transfer functions collapse in the compact limit, even if the one-dimensional wave as- 


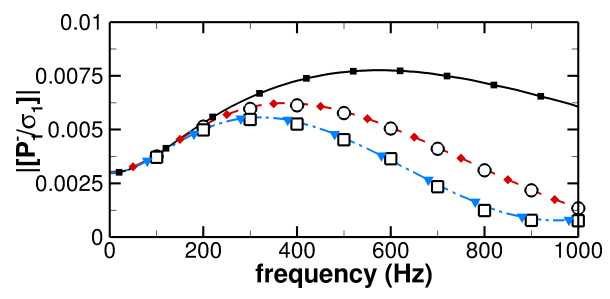

(a)

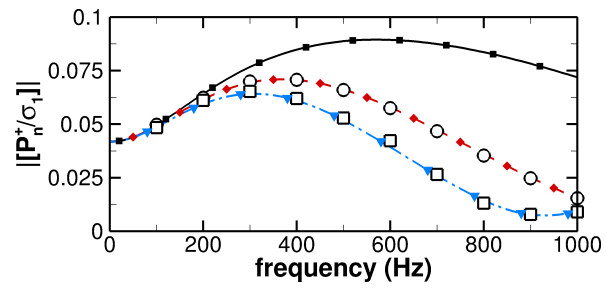

(c)

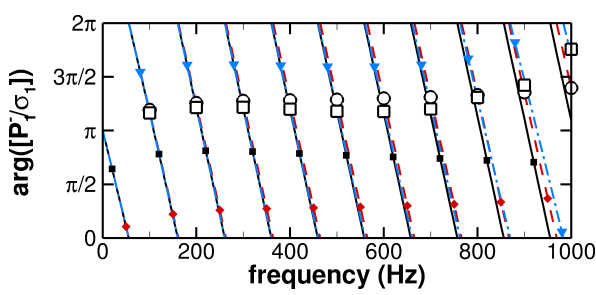

(b)

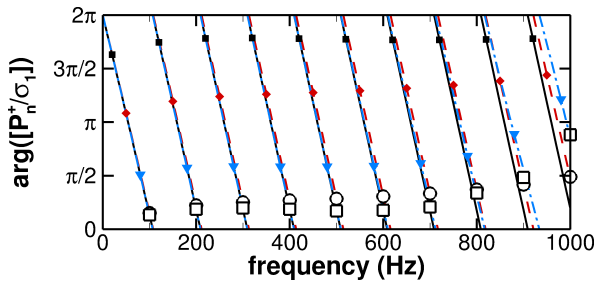

(d)

Figure 10: Computed and modelled inviscid nozzle transfer functions with entropy forcing $\sigma_{1}$.

(a) upstream wave, amplitude, (b) upstream wave, phase, (c) downstream wave, amplitude,

(d) downstream wave, phase. ॰ CFD Euler without entropy fluctuation near the wall; $\square$ CFD

Euler with entropy fluctuation near the wall; - - quasi-1D model; $--2 \mathrm{D}$ model (Euler mean flow) without entropy fluctuation near the wall; $\mathbf{\nabla}-\cdot 2 \mathrm{D}$ model (Euler mean flow) with entropy fluctuation near the wall.

sumption is not always verified for the entropy wave. Second, the quasi-1D low-order modelling strongly overestimates both numerical transfer functions for medium and large frequencies. This result has already been observed by Emmanuelli et al. 25] and is the consequence of the shear dispersion of the entropy wave through the nozzle, all the more important as frequency increases, and not reproduced with the quasi-1D assumption. When shear dispersion is taken into account in the modelling (2D model with entropy fluctuation near the wall), excellent agreement is obtained with the corresponding Euler simulation. It is also worth mentioning that no discrepancy is noticed here between the model and the simulation in the high frequency range for $\left[P_{1}^{-} / \sigma_{1}\right]$, on the contrary to what was previously observed by Emmanuelli et al. This discrepancy hence cannot come from the possible contribution of vorticity, as evoked 
in their article, but is due to numerical errors in the CAA approach used in the previous study, amplified by the low noise levels at these frequencies.

Numerically, the absence of entropy fluctuations near the wall (CFD Euler without entropy fluctuation near the wall) leads to an increase of the generated noise in both upstream and downstream directions for medium and highfrequency noise, whereas the phases of the acoustic waves do not significantly vary. These results are very well captured by the $2 \mathrm{D}$ model, which reproduces the thermo-acoustic transfer functions with an excellent agreement both in amplitude and phase. In the model, the entropy noise source terms are related to the section-averaged value of the entropy fluctuation, see Eqs. 32)-(33). As illustrated in Fig. 9 the shear dispersion of the entropy wave is very important inside the nozzle and leads to significant radial variations of the entropy fluctuations. Due to a compensation between positive and negative entropy fluctuations, the section-averaged entropy fluctuation is much lower than its initial amplitude. This is particularly visible in the converging section of the nozzle. When entropy fluctuations are removed near the nozzle wall, this compensation is reduced and the entropy noise source term locally increases in comparison to the case where the fluctuation is present up to the wall, thus leading to a more important noise production. Physically, it corresponds to a lower decorrelation of the local entropy-related noise sources in the radial direction in comparison to fully planar entropy waves. This phenomenon is very similar to that already introduced to explain differences between the quasi-1D model (high correlation along the radial direction) and the $2 \mathrm{D}$ model (low correlation).

\subsubsection{Contribution of viscosity to entropy noise}

Before discussing the simulated and modelled thermo-acoustic transfer functions, the entropy fluctuations obtained with CFD Euler and RANS approaches for a harmonic forcing at $1000 \mathrm{~Hz}$ are reproduced in Fig. 11 (a). Simulated entropy fluctuations are globally similar but two main differences may be commented. First, the shear dispersion is slightly more visible near the wall for the URANS simulation, e.g. for $x \sim 100 \mathrm{~mm}$, because this region corresponds 


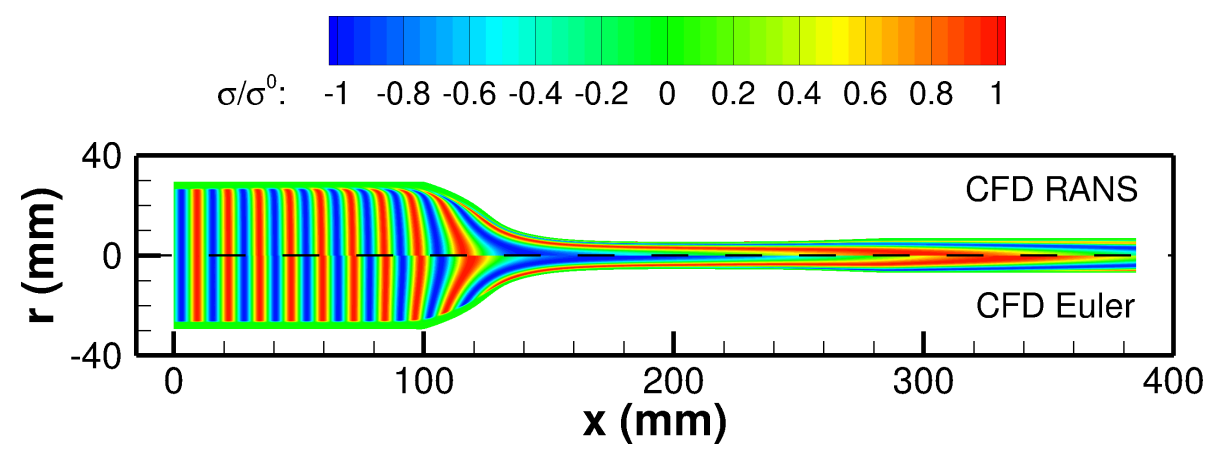

(a)

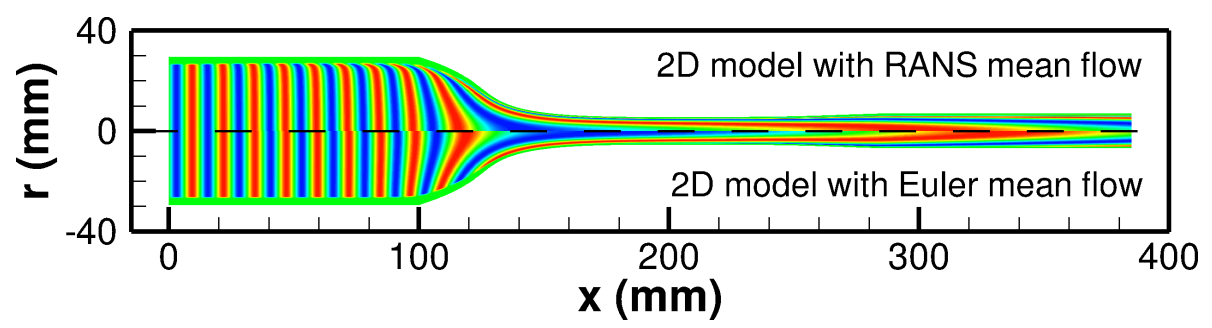

(b)

Figure 11: Entropy fluctuation at $1000 \mathrm{~Hz}$ with RANS (top) and Euler (bottom) mean flow fields obtained by (a) numerical simulations and (b) 2D modelling.

to the external part of the boundary layer with decreased velocity compared to the Euler simulation. Second, the entropy wave fronts are not in phase and appear to be convected at a lower velocity in URANS, although the same velocity is imposed outside of the boundary layer in both simulations. This is a consequence of the pressure loss. In the upstream duct, mean pressure slightly decreases along the axial direction in the URANS simulation because of viscosity. Due to the ideal gas law, and because temperature remains constant in the duct, density grows as pressure drops, so that velocity also drops for mass conservation purposes. These two effects are well reproduced by the $2 \mathrm{D}$ model and predicted entropy fluctuations along the nozzle collapse very well with the simulated ones, as illustrated in Fig. 11 (b).

The simulated and modelled thermo-acoustic transfer functions are now re- 


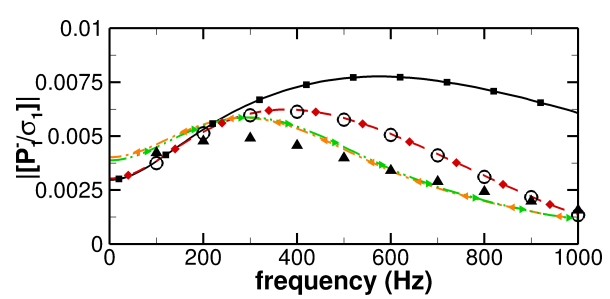

(a)

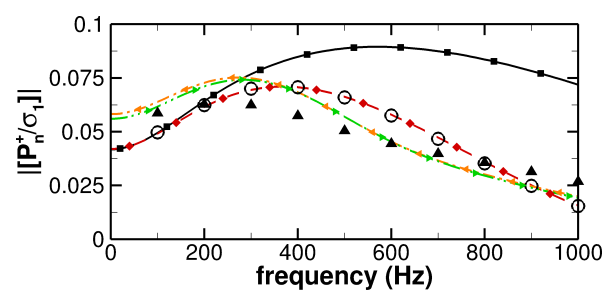

(c)

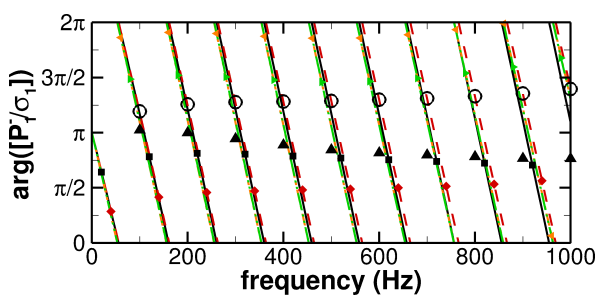

(b)

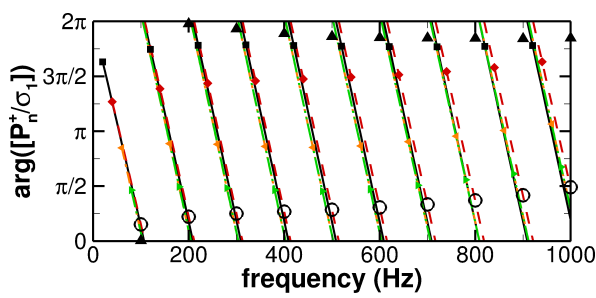

(d)

Figure 12: Computed and modelled nozzle transfer functions with entropy forcing $\sigma_{1}$. (a) upstream wave, amplitude, (b) upstream wave, phase, (c) downstream wave, amplitude, (d) downstream wave, phase. See Fig. 6 for legend.

produced in Fig. 12. Using CFD, viscosity essentially reduces the amplitude of the transfer functions, up to $-30 \%(-3 \mathrm{~dB})$ at $500 \mathrm{~Hz}$. Small noise increase is also observed for the low frequencies as well as for the high frequencies. Such low-frequency noise increase and medium-frequency noise reduction with viscous mean flow fields have already been observed numerically by Becerril 34. Lowfrequency noise increases for dimensionless convective wavelengths $\Lambda=\lambda_{\sigma} / L$ above 0.3 , where $\lambda_{\sigma}$ is the entropy wavelength in the upstream duct and $L$ the nozzle length. It is therefore limited to frequencies close to the compact limit (i.e. $\left.\lambda_{\sigma} \gg L\right)$ and, on the opposite, noise reduction occurs when noncompactness effects become important. In addition, phases are quite close for low frequencies but discrepancies rise as frequency increases. This is essentially caused by the different convection velocities of the entropy fluctuations in the upstream duct, discussed above, and is globally well reproduced by the model.

For the models, the noise modifications with viscous flow are qualitatively 
well captured. The medium-frequency noise reduction and low- and highfrequency noise increase are reproduced, even if the cross-over frequencies do not collapse with the numerical data. The agreement is nevertheless not as good as for the inviscid case and discrepancies are particularly visible in the low-frequency part of the transfer functions, over-estimated by the model. Of particular interest, no significant differences are visible for the $2 \mathrm{D}$ modelled transfer functions with RANS mean flow including or not the viscous terms in the perturbed flow equations. As previously stated for acoustic transfer functions, this means the contribution of viscous terms in Eqs. 22 - 31 is negligible for the propagation of the acoustic perturbations. It indicates in addition that modelled turbulent kinetic energy, present in the entropy noise source term of the momentum equation, Eq. (33), does not contribute in the generation of entropy noise. From a practical point of view, using the inviscid 2D model with RANS mean flow fields leads to acceptable modelled transfer functions.

Several reasons can be envisaged to explain the discrepancies between viscous numerical simulations and 2D modelled transfer functions with RANS mean flow. First, it can be argued that entropy noise sources, in particular the approximated source term associated with viscosity in Eq. (34), are not accurately reproduced analytically. The excellent agreement between the Euler simulation and the 2D inviscid model on the one side, and the 2D modelled transfer function taking or not viscous terms into account on the other side nevertheless prove that source terms are correctly captured by the model. Second, entropy fluctuations are known to generate vorticity through the baroclinic torque [24], which may in turn produce noise [50, 51]. It is reasonable to assume that the production of vorticity increases with viscosity, and as this vorticity is absent from the model, its associated noise cannot be captured. Numerical simulations nevertheless show that vorticity is of the same order for Euler and RANS simulations and therefore cannot be held responsible for the discrepancies observed. Third, it was pointed out previously that thermo-acoustic transfer functions combine the generation of entropy noise inside the nozzle and its scattering by the mean flow. The acoustic transfer functions obtained in the previous section 
evidenced that this scattering is not perfectly reproduced by the model because of the boundary layer effect. The errors associated with this scattering are of the same order as those observed for the thermo-acoustic transfer functions. The discrepancies thus seem to be a consequence of the flawed reproduction of noise scattering by the model.

\section{Conclusion}

The influence of viscosity on entropy noise generation and scattering inside a nozzle is investigated numerically and analytically. Acoustic and thermoacoustic nozzle transfer functions are evaluated numerically by solving Euler and URANS equations, whereas the inviscid analytical model of Emmanuelli et al. 25], based on the linearization of the flow equations, is extended to include viscous terms. With both approaches, the interactions between entropy and acoustic fluctuations and the mean flow field are correctly accounted for, but small scale turbulence is not taken into account and its contribution to entropy dispersion and noise scattering is not reproduced. This contribution is expected to be of minor importance in contrast with the influence of mean flow and viscous diffusion on the resolved perturbations.

Numerically, viscosity slightly modifies acoustic scattering but changes in transmission and reflection coefficients of the nozzle are limited. Such modifications are qualitatively well captured by the viscous model, even if the quantitative agreement is lower than for inviscid flows. Discrepancies are caused by the radial evolution of the acoustic waves in the thick boundary layer, not accounted for by the model that assumes planar acoustic waves. Considering entropy forcing, an important shear dispersion is first observed near the walls due to the boundary layer and dissipates entropy fluctuations. A similar dissipation is most likely to occur in real configurations and entropy fluctuations must be removed near the walls for accurate predictions. The capacity of the model to reproduce entropy noise generation in such cases is demonstrated for inviscid flows. The absence of entropy fluctuations near the nozzle wall leads to 
a noise increase, explained by the lower decorrelation of the related noise sources in the radial direction in comparison to initially planar entropy waves. Second, viscosity essentially reduces the amplitude of the transfer functions obtained by simulation in the medium frequency range. Noise reduction is reproduced by the model but discrepancies remain, as for acoustic forcing. Changes are almost totally captured when considering a viscous mean flow field, whereas viscous entropy noise source term and viscous diffusion of acoustic perturbations have a negligible impact on the noise. Discrepancies with numerical simulations are hence attributed to the inaccurate noise scattering discussed in the acoustically forced cases.

\section{Acknowledgements}

This work is funded by ONERA. The authors are grateful to Marc Terracol, François Vuillot and Lionel Matuszewski from ONERA for fruitful discussions on numerical simulations.

\section{Appendix A. Derivation of the filtered momentum equation}

The filtered momentum equation is obtained from the momentum equation, Eq. A.1) 52

$$
\frac{\partial \rho \vec{u}}{\partial t}+\vec{\nabla} \cdot(\rho \vec{u} \otimes \vec{u})=-\vec{\nabla} p+\vec{\nabla} \cdot \tau
$$

710

where $\otimes$ is the tensor product, $\tau=\mu\left(\vec{\nabla}^{T} \vec{u}+\vec{\nabla} \vec{u}\right)-2 / 3 \mu(\vec{\nabla} \cdot \vec{u}) I$ is the viscous stress tensor, $\mu$ is the dynamic viscosity of the fluid and $I$ the identity matrix, to which we apply the filter operator noted $\overline{(\cdot)}$. This operator corresponds either to the Reynolds (ensemble) averaging defined in Eq. A.2 for the RANS approach [53] or to the spatio-temporal filter of Eq. A.3. for LES [52]

$$
\begin{aligned}
& \bar{\phi}(\vec{x}, t)=\lim _{N \rightarrow+\infty} \frac{1}{N} \sum_{n=1}^{N} \phi_{n}(\vec{x}, t) \quad \text { for RANS } \\
& \bar{\phi}(\vec{x}, t)=G(\bar{\Delta}, \bar{\theta}) \star \phi(\vec{x}, t) \quad \text { for LES }
\end{aligned}
$$


with $N$ the number of realizations, $G(\bar{\Delta}, \bar{\theta})$ the kernel of the filter, $\star$ the convolution product and $\bar{\Delta}$ and $\bar{\theta}$ the cutoff length and the cutoff time, respectively. The filtered equation hence writes

$$
\frac{\partial \bar{\rho} \overrightarrow{\tilde{u}}}{\partial t}+\vec{\nabla} \cdot(\bar{\rho} \overrightarrow{\tilde{u}} \otimes \overrightarrow{\tilde{u}})=-\vec{\nabla} \bar{p}+\vec{\nabla} \cdot \bar{\tau}-\vec{\nabla} \cdot(\bar{\rho} \widetilde{\vec{u} \otimes \vec{u}}-\bar{\rho} \overrightarrow{\tilde{u}} \otimes \overrightarrow{\tilde{u}})
$$

with $\tilde{\phi}$ the Favre (mass-weighted) averaging defined from Reynolds averaging as $\tilde{\phi}=\overline{\rho \phi} / \bar{\rho}$. In Eq. A.4 , $(\widetilde{\rho} \overrightarrow{\vec{u} \otimes \vec{u}}-\bar{\rho} \overrightarrow{\vec{u}} \otimes \overrightarrow{\tilde{u}})$ needs to be modelled. The closure is classically achieved using the Boussinesq approximation with suitable generalization for compressible flows [42, 53]:

$$
-(\bar{\rho} \overrightarrow{\vec{u} \otimes \vec{u}}-\bar{\rho} \overrightarrow{\tilde{u}} \otimes \overrightarrow{\tilde{u}})=\mu_{T}\left(\vec{\nabla}^{T} \overrightarrow{\tilde{u}}+\vec{\nabla} \overrightarrow{\tilde{u}}\right)-\frac{2}{3} \mu_{T}(\vec{\nabla} \cdot \overrightarrow{\tilde{u}}) I-\frac{2}{3} \bar{\rho} k I
$$

with $\mu_{T}$ the eddy viscosity and $k$ the kinetic energy per unit volume of the modelled fluctuations. To end, the filtered viscous stress tensor is approximated by its expression evaluated from the resolved variables

$$
\bar{\tau} \approx \mu(\tilde{T})\left(\vec{\nabla}^{T} \overrightarrow{\tilde{u}}+\vec{\nabla} \overrightarrow{\tilde{u}}\right)-\frac{2}{3} \mu(\tilde{T})(\vec{\nabla} \cdot \overrightarrow{\tilde{u}}) I
$$

It has been verified numerically that this assumption is globally verified both for RANS [54, 55] and LES [42, 56] and Eq. (A.4) finally writes

$$
\frac{\partial \bar{\rho} \overrightarrow{\tilde{u}}}{\partial t}+\vec{\nabla} \cdot(\bar{\rho} \overrightarrow{\tilde{u}} \otimes \overrightarrow{\tilde{u}})=-\vec{\nabla} \bar{p}+\vec{\nabla} \cdot \bar{\tau}^{f}
$$

with

$$
\bar{\tau}^{f}=\left(1+\frac{\mu_{T}}{\mu}\right) \bar{\tau}-\frac{2}{3} \bar{\rho} k I
$$

715

It is worth noting that Eq. A.7 reduces to the initial momentum equation, used for instance in DNS, when the filter operator corresponds to identity and $\mu_{T}=k=0$.

\section{Appendix B. Simplification of the momentum-equation source term}

The momentum-equation source term writes, Eq. 33

$$
\hat{S}_{k+1 / 2}^{M}=\overline{\left(\left[u_{0 x} \frac{\partial u_{0 x}}{\partial x}+u_{0 r} \frac{\partial u_{0 x}}{\partial r}\right] \hat{\sigma}\right)}+\frac{2}{3\left(\frac{1}{\rho_{0}} \frac{\partial\left(\rho_{0} k \hat{\sigma}\right)}{\partial x}\right)}
$$




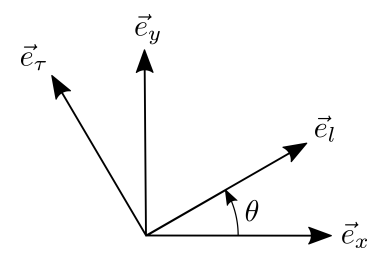

Figure B.1: Cartesian and curvilinear systems.

The second term may be developed to give

$$
\frac{1}{\rho_{0}} \frac{\partial\left(\rho_{0} k \hat{\sigma}\right)}{\partial x}=k \frac{\partial \hat{\sigma}}{\partial x}+\frac{\hat{\sigma}}{\rho_{0}} \frac{\partial\left(\rho_{0} k\right)}{\partial x}
$$

and the chain rule gives

$$
\frac{\partial \hat{\sigma}}{\partial x}=\frac{\partial \hat{\sigma}}{\partial l} \frac{\partial l}{\partial x}+\frac{\partial \hat{\sigma}}{\partial \tau} \frac{\partial \tau}{\partial x}
$$

with $l$ the curvilinear abscissa based on a streamline and $\tau$ its normal direction, see Fig. B.1. Noting that the entropy fluctuation writes analytically (see $\$ 3.4$ )

$$
\hat{\sigma}(l)=\hat{\sigma}(l=0) \exp \left(-\mathrm{i} \omega \int_{0}^{l} \frac{\mathrm{d} \zeta}{u_{0}(\zeta)}\right)
$$

the derivative along the $l$-direction may be further simplified

$$
\frac{\partial \hat{\sigma}}{\partial l}=-\frac{i \omega}{u_{0}} \hat{\sigma}
$$

The derivative along the $\tau$-direction may be neglected when the flow incidence $\theta$ is small (generally verified in the diffuser, but possibly too restrictive in the convergent section of the nozzle), so that the source term may be approximated by

$$
\hat{S}_{k+1 / 2}^{M} \simeq \overline{\left(\left[u_{0 x} \frac{\partial u_{0 x}}{\partial x}+u_{0 r} \frac{\partial u_{0 x}}{\partial r}\right] \hat{\sigma}\right)}+\frac{2}{3\left(\left[-\frac{\mathrm{i} \omega k}{u_{0}} \frac{\partial l}{\partial x}+\frac{1}{\rho_{0}} \frac{\partial\left(\rho_{0} k\right)}{\partial x}\right] \hat{\sigma}\right)}
$$

with $\partial l / \partial x=1 / \cos \theta$ and $\tan \theta=u_{0 r} / u_{0 x}$ by definition.

To comply with the formalism used in CHEOPS-Nozzle, which is necessary to take into account the presence of a normal shock in the diffuser [26], the source term is rewritten

$$
\hat{S}_{k+1 / 2}^{M}=\sum_{j=1}^{n j} \nu_{k+1 / 2}^{j} \hat{\sigma}_{j}^{0}
$$


720

$$
\begin{aligned}
\nu_{l+1 / 2}^{j}=\left(\frac{A_{j}}{A}[\right. & u_{0 x, j} \frac{\partial u_{0 x, j}}{\partial x}+u_{0 r, j} \frac{\partial u_{0 x, j}}{\partial r} \\
& \left.\left.+\frac{2}{3}\left(-\frac{\mathrm{i} \omega k_{j}}{u_{0, j}}\left(\frac{\partial l}{\partial x}\right)_{j}+\frac{1}{\rho_{0, j}} \frac{\partial \rho_{0, j} k_{j}}{\partial x}\right)\right]\right)_{l+1 / 2} e^{i \varphi_{l+1 / 2, j}}(\mathrm{~B} .8)
\end{aligned}
$$

where $n j$ is the number of streamtubes and $A_{j}$ the section of the $j^{\text {th }}$ streamtube.

[1] A. P. Dowling, Y. Mahmoudi, Combustion noise, Proc. Combust. Inst. 35 (2015) 65-100. doi:https://doi.org/10.1016/j.proci.2014.08.016

[2] M. Ihme, Combustion and engine-core noise, Annu. Rev. Fluid

$$
\text { Mech. } 49 \text { (2017) 277-310. doi:https://doi.org/10.1146/ }
$$
annurev-fluid-122414-034542.

[3] D. Blacodon, S. Lewy, Source localization of turboshaft engine broadband noise using a three-sensor coherence method, J. Sound Vib. 338 (2015) 250-262. doi:https://doi.org/10.1016/j.jsv.2014.10.019.

${ }_{730}^{2}$ [4] T. Livebardon, S. Moreau, L. Gicquel, T. Poinsot, E. Bouty, Combining LES of combustion chamber and an actuator disk theory to predict combustion noise in a helicopter engine, Combust. Flame 165 (2016) 272-287. doi:https://doi.org/10.1016/j.combustflame.2015.12.012.

[5] W. C. Strahle, Some results in combustion generated noise, J. Sound 735

Vib. 23 (1972) 113-125. doi:https://doi.org/10.1016/0022-460X (72) 90792-4.

[6] H. Hassan, Scaling of combustion generated noise, J. Fluid Mech. 49 (1974) 445-453. doi:https://doi.org/10.1017/S0022112074000292.

[7] S. Candel, Analytical studies of some acoustic problems of jet engines, Ph.D. thesis, California Institute of Technology (1972).

[8] F. E. Marble, Acoustic disturbance from gas non-uniformities convecting through a nozzle, in: Proceedings of the Interagency Symposium on Uni- 
versity Research in Transportation Noise, Stanford University, California, pp. 547-561, 1973.

[9] C. L. Morfey, Amplification of aerodynamic noise by convected flow inhomogeneities, J. Sound Vib. 31 (1973) 391-397. doi:https://doi.org/10. 1016/S0022-460X (73)80255-X.

[10] L. Magri, On indirect noise in multicomponent nozzle flows, J. Fluid Mech. 828 (2017) R2. doi:https://doi.org/10.1017/jfm.2017.591.

[11] C. K. W. Tam, S. A. Parrish, The physical processes of indirect combustion noise generation, Int. J. Aeroacoust. 17 (2018) 22-35. doi:https://doi. org/10.1177/1475472X17743628.

[12] C. K. W. Tam, F. Bake, L. S. Hultgren, T. Poinsot, Combustion noise: modeling and prediction, CEAS Aeronaut. J. 10 (2019) 101-122. doi: https://doi.org/10.1007/s13272-019-00377-2.

[13] W. C. Strahle, On combustion generated noise, J. Fluid Mech. 49 (1971) 399-414. doi:https://doi.org/10.1017/S0022112071002167.

[14] M. Leyko, F. Nicoud, T. Poinsot, Comparison of direct and indirect combustion noise mechanisms in a model combustor, AIAA J. 47 (2009) 27092716. doi:https://doi.org/10.2514/1.43729.

[15] A. S. Morgans, I. Duran, Entropy noise: A review of theory, progress and challenges, Int. J. Spay Combust. Dyn. 8 (4) (2016) 285-298. doi:https: //doi.org/10.1177/1756827716651791.

[16] L. Magri, J. O'Brien, M. Ihme, Compositional inhomogeneities as a source 765 of indirect combustion noise, J. Fluid Mech. 799 (2016) R4. doi:https: //doi.org/10.1017/jfm.2016.397

[17] L. Magri, J. O'Brien, M. Ihme, Effects of nozzle Helmholtz number on indirect combustion noise by compositional perturbations, J. Eng. Gas Turbine Power 140 (2018) 031501. doi:https://doi.org/10.1115/1.4037914. 
[19] W. H. Moase, M. J. Brear, C. Manzie, The forced response of choked nozzles

[20] A. Giauque, M. Huet, F. Clero, Analytical analysis of indirect combustion noise in subcritical nozzles, J. Eng. Gas Turbine Power 134 (2012) 111202. doi:https://doi.org/10.1115/1.4007318

[21] M. S. Bohn, Response of a subsonic nozzle to acoustic and entropy dis-

[25] A. Emmanuelli, J. Zheng, M. Huet, A. Giauque, T. Le Garrec, S. Ducruix, Description and application of a 2D-axisymmetric model for entropy noise convected through a nozzle, J.Sound Vib. 55 (1977) 225-243. doi:https: //doi.org/10.1016/0022-460X (77)90596-X and supersonic diffusers, J. Fluid Mech. 585 (2007) 281-304. doi:https: //doi.org/10.1017/S0022112007006647. turbances, J. Sound Vib. 52 (1977) 283-297. doi:https://doi.org/10. 1016/0022-460X (77)90647-2.

[22] I. Duran, S. Moreau, Solution of the quasi-one-dimensional linearized euler equations using flow invariants and the Magnus expansion, J. Fluid Mech. 723 (2013) 190-231. doi:https://doi.org/10.1017/jfm.2013.118

[23] Y. Mahmoudi, A. P. Dowling, S. R. Stow, Acoustic and entropy waves in nozzles in combustion noise framework, AIAA J. 55 (2017) 2369-2381. doi:https://doi.org/10.2514/1.J055597.

[24] I. Duran, A. S. Morgans, On the reflection and transmission of circumferential waves through nozzles, J. Fluid Mech. 773 (2015) 137-153. doi:https://doi.org/10.1017/jfm.2015.247. in nozzle flows, J. Sound Vib. 472 (2020) 115163. doi:https://doi.org/ $10.1016 / j \cdot j s v .2019 .115163$

[26] M. Huet, A. Emmanuelli, T. Le Garrec, Entropy noise modelling in 2D choked nozzle flows, J. Sound Vib. 488 (2020) 115637. doi:https://doi. org $/ 10.1016 / j \cdot j$ sv.2020.115637. 
[27] Y. Mahmoudi, A. Giusti, E. Mastorakos, A. P. Dowling, Low-order modeling of combustion noise in an aero-engine: The effect of entropy dispersion, J. Eng. Gas Turbines and Power 140 (2018) 011502. doi:https: //doi.org/10.1115/1.4037321.

[28] F. De Domenico, E. O. Rolland, S. Hochgreb, A generalised model for acoustic and entropic transfer function of nozzles with losses, J. Sound Vib. 440 (2019) 212-230. doi:https://doi.org/10.1016/j.jsv.2018.

[29] M. Huet, Budgets of disturbances energy for nozzle flows at subsonic and choked regimes, J. Eng. Gas Turbine Power 140 (2018) 112602. doi:https: //doi.org/10.1115/1.4038473.

[30] M. Leyko, S. Moreau, F. Nicoud, T. Poinsot, Numerical and analytical modelling of entropy noise in a supersonic nozzle with a shock, J. Sound Vib. 330 (2011) 3944-3958. doi:https://doi.org/10.1016/j.jsv.2011. 01.025

[31] F. Bake, C. Richter, B. Mühlbauer, N. Kings, I. Röhle, F. Thiele, B. Noll, The Entropy Wave Generator (EWG): A reference case on entropy noise, J.

815 Sound Vib. 326 (2009) 574-598. doi:https://doi.org/10.1016/j.jsv. 2009.05 .018

[32] A. S. Morgans, C. S. Goh, J. A. Dahan, The dissipation and shear dispersion of entropy waves in combustor thermoacoustics, J. Fluid Mech. 733 (2013) R2. doi:https://doi.org/10.1017/jfm.2013.448,

[33] A. Giusti, N. A. Worth, E. Mastorakos, A. P. Dowling, Experimental and numerical investigation into the propagation of entropy waves, AIAA J. 55 (2017) 446-458. doi:https://doi.org/10.2514/1.J055199.

[34] C. Becerril Aguirre, Simulation of noise emitted by a reactive flow, Ph.D. thesis, Institut National Polytechnique de Toulouse (2017). 

of indirect combustion noise in the entropy wave generator experiment, Int.

J. Spay Combust. Dyn. 10 (2018) 154-168. doi:https://doi.org/10. $1177 / 1756827717740775$.

[36] Y. Xia, I. Duran, A. S. Morgans, X. Han, Dispersion of entropy perturbations transporting through an industrial gas turbine combustor, Flow Turbul. Combust. 100 (2018) 481-502. doi:https://dx.doi.org/10.1007/ s10494-017-9854-6.

[37] M. Huet, Influence of calorically perfect gas assumption and thermal diffusion on indirect noise generation, in: 24th International Congress on Sound and Vibration, London, United Kingdom, 2017.

[38] W. C. Ullrich, M. Schulze, T. Sattelmayer, Fundamental indirect noise generation by interactions between entropy, vorticity and acoustic waves in the context of aero engine applications, in: 43rd International Congress on Noise Control Engineering, Melbourne, Australia, 2014.

[39] J.-M. Lourier, A. Huber, B. Noll, M. Aigner, Numerical analysis of indirect combustion noise generation within a subsonic nozzle, AIAA J. 52 (2014) 2114-2126. doi:https://doi.org/10.2514/1.J052755.

[40] T. Bach, Y. Mahmoudi, S. Spence, Unsteady RANS simulation on the effect of film cooling on entropy noise generation in a two-dimensional stator cascade, in: Proceedings of the The 26th International Congress on Sound and Vibration, 2019.

[41] L. Pinelli, L. Lilli, A. Arnone, P. Gaetani, G. Persico, Numerical study of entropy wave evolution within a HPT stage, in: Proceedings of the 75 th ATI Congress, 2020.

[42] J. Blazek, Computational Fluid Dynamics: Principles and Applications,

घ 2nd Edition, Elsevier, Oxford, 2005. doi:https://doi.org/10.1016/ B978-0-08-044506-9.X5000-0. 
[43] K. A. Hoffman, S. T. Chiang, S. Saddiqui, M. Papadakis, Fundamental equations of fluid mechanics, Engineering Education System, Wichita, Kansas, 1996.

[44] A. Refloch, B. Courbet, A. Murrone, P. Villedieu, C. Laurent, P. Gilbank, J. Troyes, L. Tessé, G. Chaineray, J. B. Dargaud, E. Quémerais, F. Vuillot, CEDRE software, Aerospace Lab 2.

[45] A. Emmanuelli, Numerical simulation and modelling of entropy noise in nozzle and turbine stator flows, Ph.D. thesis, Université Paris-Saclay (2019).

[46] P. Guillaume, J. Schoukens, R. Pintelon, I. Kollár, Crest-factor minimization using nonlinear chebyshev approximation methods, IEEE Trans. Instrum. Meas. 40 (1991) 982-989. doi:https://doi.org/10.1109/19. 119778

[47] J. Zheng, Analytical and numerical study of the indirect combustion noise generated by entropy disturbances in nozzle flows, $\mathrm{Ph} . \mathrm{D}$. thesis, Université Paris-Saclay (2016).

[48] C. Bogey, S. Barré, C. Bailly, Direct computation of the noise generated by subsonic jets originating from a straight pipe nozzle, Int. J. Aeroacoust. 7 (2008) 1-22. doi:https://doi.org/10.1260/147547208784079917.

[49] J. Kopitz, E. Bröcker, W. Polifke, Characteristics-based filter for identification of planar acoustic waves in numerical simulation of turbulent compressible flow, in: 12th International Congress on Sound and Vibration, $875 \quad$ Lisbon, Portugal, 2005.

[50] N. A. Cumpsty, F. E. Marble, The interaction of entropy fluctuations with turbine blade rows; a mechanism of turbojet engine noise, Proc. Math. Phys. Eng. Sci. 357 (1977) 323-344. doi:https://doi.org/10.1098/ rspa.1977.0171. 
[51] N. Kings, F. Bake, Indirect combustion noise: noise generation by accelerated vorticity in a nozzle flow, Int. J. Spray Combust. Dyn. 2 (2010) 253-266. doi:https://doi.org/10.1260/1756-8277.2.3.253.

[52] C. Wagner, T. Hüttl, P. Sagaut (Eds.), Large-Eddy Simulation for Acoustics, Cambridge University Press, New York, 2007.

[53] D. C. Wilcox, Turbulence Modeling for CFD, 3rd Edition, DCW Industries, La Cañada, California, 2006.

[54] S. Barre, J.-P. Bonnet, T. B. Gatski, N. D. Sandham, Compressible, high speed flows, in: B. E. Launder, N. D. Sandham (Eds.), Closure Strategies for Turbulent and Transitional Flows, Cambridge University Press, Cambridge, 2002, Ch. 19, pp. 522-581. doi:https://doi.org/10.1017/ CB09780511755385.021.

[55] M. Rai, T. Gatski, G. Erlebacher, Direct simulation of spatially evolving compressible turbulent boundary layers, in: Proceedings of the 33rd Aerospace Sciences Meeting and Exhibit, Paper AIAA 1995-583, Reno, Nevada, 1995. doi:https://doi.org/10.2514/6.1995-583.

[56] B. Vreman, B. Geurts, H. Kuerten, A priori tests of large eddy simulation of the compressible plane mixing layer, J. Eng. Math. 29 (1995) 299-327. doi:https://doi.org/10.1007/BF00042759. 